\title{
Diffusion as a singular homogenization of the Frenkel-Kontorova model
}

\author{
N. Alibaud $d^{*,}$, A. Briani ${ }^{\ddagger}$, R. Monneau ${ }^{\S}$
}

May 15, 2014

\begin{abstract}
In this work, we consider a general fully overdamped Frenkel-Kontorova model. This model describes the dynamics of a infinite chain of particles, moving in a periodic landscape. Our aim is to describe the macroscopic behavior of this system. We study a singular limit corresponding to a high density of particles moving in a vanishing periodic landscape. We identify the limit equation which is a nonlinear diffusion equation. Our homogenization approach is done in the framework of viscosity solutions.
\end{abstract}

AMS Classification: 35B27, 40L25, 35B10.

Keywords: particle systems, periodic homogenization, Frenkel-Kontorova models, Hamilton-Jacobi equations, nonlinear diffusion.

\section{Introduction}

In this paper we consider the dynamics of particles on the real line, moving with interactions with their neighbors and with a periodic landscape. For each $i \in \mathbb{Z}$, the position of the $i$-th particle is called $U_{i}(\tau) \in \mathbb{R}$, where $\tau$ is the time. These particles are assumed to solve a generalized fully overdamped Frenkel-Kontorova (FK) model. In this model, the velocity is proportional to the force acting on the particles. The classical physical model was introduced by Kontorova, Frenkel in [12] to describe the plasticity at a microscopic level. For a good overview on the FK model and on its applications, we refer to the recent book [4] of Braun and Kivshar and the article of Floria and Mazo [7]. In the present paper, our generalized fully overdamped FK model is the following infinite system of ODEs:

$$
\begin{cases}\frac{d U_{i}}{d \tau}(\tau)=\varepsilon^{2(\alpha-1)} F\left(\left[\frac{U_{i+j}(\tau)-U_{i}(\tau)}{\varepsilon^{2(\alpha-1)}}\right]_{j}^{m}, U_{i}(\tau)\right) & \text { for } \tau \in(0, \infty) \text { and } i \in \mathbb{Z} \\ U_{i}(0)=\frac{1}{\varepsilon} u_{0}\left(i \varepsilon^{\alpha}\right) & \text { for } i \in \mathbb{Z},\end{cases}
$$

\footnotetext{
*Laboratoire de Mathématiques de Besançon, UMR CNRS 6623, 16 route de Gray, 25030 Besançon cedex, France.

${ }^{\dagger}$ Department of Mathematics, Faculty of Science, Prince of Songkla University, Hat Yai, Songkhla, Thailand, 90112 .

${ }^{\ddagger}$ Dipartimento di Matematica, Universitá di Pisa, Largo B. Pontecorvo 5, 56127 Pisa Italia; UMA, ENSTA, 32 Boulevard Victor, 75739 Paris cedex 15 Paris cedex 15, France.

${ }^{\S}$ CERMICS, Paris Est-ENPC, 6 and 8 avenue Blaise Pascal, Cité Descartes, Champs sur Marne, 77455, Marne la Vallée Cedex 2, France.
} 
where $\varepsilon>0$ is a small parameter and we use the notation

$$
\left[V_{j}\right]_{j}^{m}=V=\left(V_{-m}, V_{-m+1}, \ldots, V_{-1}, V_{1}, \ldots, V_{m-1}, V_{m}\right) \in \mathbb{R}^{2 m}
$$

for some fixed $m \in \mathbb{N}^{*}$. This dynamics corresponds to general interactions with the $m$ nearest neighbors on the left and the $m$ nearest neighbors on the right.

Our goal is to study the macroscopic behaviour of the chain of particles as $\varepsilon$ goes to zero. We use the following assumptions on the initial data $u_{0}$, on the interactions $F$ and on the exponent $\alpha$.

(H0) (Initial gradient bounded from below and above)

The function $u_{0}$ is such that $\left(u_{0}\right)_{x} \in W^{2, \infty}(\mathbb{R})$ and

$$
\delta_{0} \leq\left(u_{0}\right)_{x} \leq \frac{1}{\delta_{0}} \quad \text { on } \quad \mathbb{R},
$$

for some constant $\delta_{0}>0$.

(H1) (i) (Regularity)

$$
F \in C^{1}\left(\mathbb{R}^{2 m} \times \mathbb{R}\right) \text { and is globally Lipschitz continuous on } \mathbb{R}^{2 m} \times \mathbb{R} \text {. }
$$

(ii) (Periodicity)

$$
\text { For all }\left(V, V_{0}\right) \in \mathbb{R}^{2 m} \times \mathbb{R}, \quad F\left(V, V_{0}+1\right)=F\left(V, V_{0}\right) .
$$

(iii) (Monotonicity)

$$
\text { For all } 1 \leq|i| \leq m, \quad F_{i}:=\frac{\partial F}{\partial V_{i}} \geq 0 \quad \text { on } \quad \mathbb{R}^{2 m} \times \mathbb{R} .
$$

(iv) (Invariance by linear additions)

$$
\text { For all }\left(V, V_{0}, q\right) \in \mathbb{R}^{2 m} \times \mathbb{R} \times \mathbb{R}, \quad F\left(\left[V_{j}+j q\right]_{j}^{m}, V_{0}\right)=F\left(\left[V_{j}\right]_{j}^{m}, V_{0}\right) .
$$

(v) (Non-degeneracy w.r.t. quadratic displacements)

$$
\text { For all }\left(V_{0}, q\right) \in \mathbb{R} \times \mathbb{R}, \quad \frac{d}{d q} F\left(\left[\frac{j^{2}}{2} q\right]_{j}^{m}, V_{0}\right) \geq \nu,
$$

for some positive constant $\nu$.

(vi) (Zero mean value of $f$ )

For all $h \in \mathbb{R}$, define $f(h)$ as the unique real such that

$$
F\left(\left[-\frac{j^{2}}{2} f(h)\right]_{j}^{m}, h\right)=0 ;
$$

the 1-periodic function $f: \mathbb{R} \rightarrow \mathbb{R}$ thus defined is assumed to satisfy

$$
\int_{0}^{1} f(h) d h=0 .
$$


(H2) (The exponent $\alpha$ ) We suppose $\alpha>2$.

Let us make some comments on these assumptions.

In assumption (H0) the relevant information is the boundedness of the gradient. The regularity of $u_{0}$ can be weakened to Lipschitz continuous by standard approximation arguments. Moreover, the regularity of the Hamiltonian $F$ in (H1) (i) can also be weakened to only $C^{1}$ (without global Lipschitz continuity). For the sake of simplicity in the proof of our main result (Theorem 1.1 below) we decide to ask for $\left(u_{0}\right)_{x}$ in $W^{2, \infty}(\mathbb{R})$ and $F$ globally Lipschitz continuous.

Let us now discuss assumption (H1). First, assumption (ii) takes into account the existence of the periodic landscape. Second, the monotonicity assumption (iii) will play a crucial role in our analysis: this will guarantee the existence of a comparison principle for our system of particles. Remark also that assumption (vi) is always satisfied for $F$ replaced by $a+F$ for a suitable constant $a \in \mathbb{R}$. The zero mean value property of the function $f$ means that the periodic landscape is balanced and does not induce any drift in the motion of the particles. The most striking condition is assumption (iv) which is technically used to remove the contribution of linear displacements of the particles, and to focus on quadratic displacements. This assumption is for instance always satisfied if $F\left(V, V_{0}\right)$ is a function depending only on the symmetric part $V_{-j}+V_{j}$ for each $j=1, \ldots, m$ and on $V_{0}$. Assumption (iv) is also satisfied if $F\left(V, V_{0}\right)$ is linear in $V$, with suitable coefficients vanishing the linear contributions $[j q]_{j}^{m}$. The non-degeneracy assumption w.r.t. the quadratic contributions $\left[\frac{j^{2}}{2} q\right]_{j}^{m}(\mathbf{v})$ is used to ensure the existence of the periodic potential $f$ by the inverse function theorem. In particular, our analysis would remain true if $\nu$ depends on $\left(V_{0}, q\right)$ and if we are sure that equation (1.2) admits at least one solution.

As far as (H2) is concerned, let us mention that one also investigates the limit case $\alpha=2$. We shall see that in this case the homogenized equation could be different. This seems to be a remarkable feature that will be discussed in a more detailled way in the sequel. The case $\alpha<2$ remains completely open.

The classical (and simplest) example of the fully overdamped Frenkel Kontorova model is

$$
\frac{d U_{i}}{d \tau}=U_{i+1}+U_{i-1}-2 U_{i}+\varepsilon^{2(\alpha-1)} \sin \left(2 \pi U_{i}\right)
$$

corresponding to interactions with the first nearest neighbors with $m=1$ and

$$
F\left(V_{-1}, V_{1}, V_{0}\right)=V_{-1}+V_{1}+f\left(V_{0}\right)
$$

with $f\left(V_{0}\right)=\sin \left(2 \pi V_{0}\right)$. Remark that for an initial datum

$$
U_{i}(0)=\frac{1}{\varepsilon} u_{0}\left(i \varepsilon^{\alpha}\right),
$$

we see that the high density of particles behaves like $\varepsilon^{1-\alpha} \frac{1}{\left(u_{0}\right)_{x}}$. This shows that our scaling corresponds to the case where the small amplitude $\varepsilon^{2(\alpha-1)}$ of the periodic potential behaves exactly as the square of the inverse of the particle density. This can be rephrased, saying that we are interested in a distinguished limit with a weak periodic landscape and height density particles.

To study the macroscopic behavior of the system in the limit as $\varepsilon$ goes to zero, it is convenient to introduce a parabolic rescaling, defining the function

$$
U^{\varepsilon}(t, x):=\varepsilon U_{\left\lfloor\frac{x}{\varepsilon^{\alpha}}\right\rfloor}\left(\frac{t}{\varepsilon^{2 \alpha}}\right)
$$


where $\lfloor\cdot\rfloor$ denotes the floor integer part. Note that if $U$ is a solution of system (1.1) then $U^{\varepsilon}$ has to solve the following equation:

$$
\begin{cases}U_{t}^{\varepsilon}(t, x)=\frac{1}{\varepsilon} F\left(\left[\frac{U^{\varepsilon}\left(t, x+j \varepsilon^{\alpha}\right)-U^{\varepsilon}(t, x)}{\varepsilon^{2 \alpha-1}}\right]_{j}^{m}, \frac{U^{\varepsilon}(t, x)}{\varepsilon}\right) & \text { for }(t, x) \in(0, \infty) \times \mathbb{R} \\ U^{\varepsilon}(0, x)=u_{0}\left(\left\lfloor\frac{x}{\varepsilon^{\alpha}}\right\rfloor \varepsilon^{\alpha}\right) & \text { for } x \in \mathbb{R},\end{cases}
$$

which will be understood in the viscosity sense.

Our first main result is the following.

\section{Theorem 1.1. (Diffusive limit by homogenization)}

Assume (H0), (H1) and (H2). There exist a unique viscosity solution $U^{\varepsilon}$ of $(1.5)$, and a function $G:(0, \infty) \rightarrow \mathbb{R}$ that does not depend on $u_{0}$ and such that

$$
G \in C^{0}(0, \infty) \text { is positive, } \lim _{p \rightarrow 0} G(p)=0 \text { and } \lim _{p \rightarrow \infty} G(p)=l>0 .
$$

Moreover, the function $U^{\varepsilon}$ converges to $u^{0}$ locally uniformly on compact sets of $[0, \infty) \times \mathbb{R}$ as $\varepsilon \rightarrow 0$, where $u^{0}$ is the unique viscosity solution of

$$
\begin{cases}u_{t}^{0}=G\left(u_{x}^{0}\right) u_{x x}^{0} & \text { for }(t, x) \in(0, \infty) \times \mathbb{R} \\ u^{0}(0, x)=u_{0}(x) & \text { for } x \in \mathbb{R} .\end{cases}
$$

Remark 1.2. Recall that for discontinuous viscosity solutions the uniqueness of solutions means the uniqueness of the semicontinuous envelopes of the solution (for further details see the proof of Theorem 2.6).

Remark that if we introduce the inverse $g$ of $u^{0}$, defined by $u^{0}(t, g(t, y))=y$, then the rescaled particle density $\rho(t, y)=g_{y}(t, y)$ solves formally the following nonlinear diffusion equation $\rho_{t}=$ $\left(\frac{1}{\rho^{2}} G\left(\frac{1}{\rho}\right) \rho_{y}\right)_{y}$. This shows a very small diffusion coefficient for large densities of particles, and a huge diffusion coefficient for small rescaled densities.

Theorem 1.1 is an homogenization result and is obtained in the framework of viscosity solutions. Let us mention the pioneering work of Lions, Papanicolau and Varadan [13] where homogenization of Hamilton-Jacobi equations has been done for the first time.

In Theorem 1.1, the effective equation satisfied by the limit is parabolic and is naturally associated to the parabolic rescaling (1.4). This result has to be compared to the homogenization result given in Forcadel, Imbert, Monneau [8] for an hyperbolic scaling (in the case $\alpha=1$ ), where the limit equation is a first order Hamilton-Jacobi equation. We can also refer the interested reader to [9], where a study of an overdamped dynamics of particles with two-body interactions is done, with particular applications to dislocation dynamics.

The main idea of the proof of Theorem 1.1 is that the solution $U^{\varepsilon}$ is locally - close to a point $\left(t_{0}, x_{0}\right)$ - well approximated by the following ansatz

$$
U^{\varepsilon}(t, x)=\varepsilon h\left(\frac{\tilde{U}^{\varepsilon}(t, x)}{\varepsilon}, u_{x}^{0}(t, x)\right) \quad \text { with } \quad \tilde{U}^{\varepsilon}(t, x):=u^{0}(t, x)+\varepsilon^{2} v\left(\frac{u^{0}(t, x)}{\varepsilon}\right),
$$

where $u^{0}$ is the limit solution, $h$ is a hull function and $v$ is a corrector. Our proof follows the lines of the "perturbed test function" method introduced by Evans in [6], based on the ansatz (1.8).

The functions $h$ and $v$ are defined below: 
Proposition 1.3. (Hull function, corrector and diffusion coefficient) Assume (H1).

\section{(a) The hull function $h$}

There exists a unique function $h: \mathbb{R} \times(0, \infty) \rightarrow \mathbb{R}$ such that $h$ is $C^{2}$ w.r.t. its first variable and satisfies for all $(z, p) \in \mathbb{R} \times(0, \infty)$,

$$
\left\{\begin{array}{l}
F\left(\left[\frac{j^{2}}{2} p^{2} h_{z z}(z, p)\right]_{j}^{m}, h(z, p)\right)=0, \\
h(z+1, p)=h(z, p)+1, \\
h_{z}(z, p)>0, \\
h(0, p)=0 .
\end{array}\right.
$$

Moreover, we have $h \in C^{3}(\mathbb{R} \times(0, \infty))$.

(b) The corrector $v$

For all $(z, p) \in \mathbb{R} \times(0, \infty)$, define

$$
A(z, p):=\frac{1}{2}+p \frac{h_{p z}(z, p)}{h_{z}(z, p)} \quad \text { and } \quad K(z, p):=\sum_{1 \leq|i| \leq m} i^{2} F_{i}\left(\left[\frac{j^{2}}{2} p^{2} h_{z z}(z, p)\right]_{j}^{m}, h(z, p)\right) .
$$

Then, for all $p_{0}>0$ and $M_{0} \in \mathbb{R}$ there exists a unique $\lambda \in \mathbb{R}$ such that there exists $v \in C^{2}(\mathbb{R})$ satisfying

$$
\begin{cases}\lambda=K\left(z, p_{0}\right) A\left(z, p_{0}\right) M_{0}+K\left(z, p_{0}\right) p_{0}^{2}\left(\frac{v_{z z}(z)}{2}+\frac{h_{z z}\left(z, p_{0}\right)}{h_{z}\left(z, p_{0}\right)} v_{z}(z)\right) & \text { for } z \in \mathbb{R} \\ v(z+1)=v(z) & \text { for } z \in \mathbb{R} .\end{cases}
$$

(c) The diffusion coefficient $G$

Finally, we have $\lambda=G\left(p_{0}\right) M_{0}$ where $G$ is the function in (1.7) and is defined for $p>0$ by

$$
G(p):=\frac{\int_{0}^{1} A(z, p) h_{z}^{2}(z, p) d z}{\int_{0}^{1} \frac{h_{z}^{2}(z, p)}{K(z, p)} d z} .
$$

In the ansatz (1.8) around the point $\left(t_{0}, x_{0}\right)$, we use the definition of $v$ given in Proposition 1.3 with $p_{0}=u_{x}^{0}\left(t_{0}, x_{0}\right)$ and $M_{0}=u_{x x}^{0}\left(t_{0}, x_{0}\right)$. Remark also that the expression of the diffusion coefficient $G$ in (1.12) is explicit, which can be underlined, in comparison to general homogenization results, where usually the effective Hamiltonian is not explicit.

Let us now list the main properties of $G$.

Proposition 1.4. (Qualitative properties of $G$ )

Assume (H1). Then, the function $G$ defined by (1.12) fulfills (1.6). Moreover $G$ satisfies: 
(a) The limit l can be computed explicitly as follows:

$$
l:=\frac{1}{2}\left\{\int_{0}^{1}\left[\sum_{1 \leq|i| \leq m} i^{2} F_{i}\left(-\left[\frac{j^{2}}{2} f(z)\right]_{j}^{m}, z\right)\right]^{-1} d z\right\}^{-1}
$$

( $f$ being the 1-periodic function in (1.2)).

(b) For each $k \in \mathbb{N}$, if $F \in C^{k+1}\left(\mathbb{R}^{2 m} \times \mathbb{R}\right)$ then $G \in C^{k}(0, \infty)$.

In general, we do not know if $G$ is a monotone function of $p$. Nevertheless, for instance in the special case of the classical FK model, we have the following additional result:

\section{Proposition 1.5. (Monotonicity of $G$ for the classical FK)}

For the classical FK model given in (1.3) with $f \in C^{1}(\mathbb{R})$, 1-periodic and with zero mean value, the function $G$ defined in (1.12) is analytic on $(0, \infty)$ and non-decreasing.

After finishing this work, we were aware of the work of Jerrard [11], where he studies general homogenization problems. A special case of his results concerns the homogenization of the particular equation

$$
u_{t}^{\varepsilon}(t, x)=u_{x x}^{\varepsilon}(t, x)+\frac{1}{\varepsilon} f\left(\frac{u^{\varepsilon}}{\varepsilon}\right) \quad \text { for } \quad(t, x) \in(0, \infty) \times \mathbb{R},
$$

with $f$ periodic with zero mean value. This model is the continuous analogue of the classical FK model with $F$ given by (1.3).

It turns out that the hull function and the corrector found by Jerrard are very similar to ours (compare equations (2.1)-(2.5) in [11] with (1.9)-(1.11) in the special case where $F$ is given by (1.3)). Moreover, by the implicit function theorem we lead our study of the hull function to the study of the correctors done in [11]. However, we decide to give here the detailed proof of Proposition 1.3 for the sake of self-consistency.

Naturally, the limit equation found in [11] is the first equation in (1.7) (i.e. $u_{t}^{0}=G\left(u_{x}^{0}\right) u_{x x}^{0}$ ), where the function $G$ is proved to fulfill properties (1.6). Therefore, as a byproduct, Proposition 1.5 shows also that the diffusion coefficient $G(p)$ found in [11] is monotone in $p$.

Let us now emphasize that for continuous PDEs as (1.14), the homogenized equation does not change if one takes $\alpha \in[1,2]$. A new phenomenon could be observed for the discrete model (1.1) as stated by our last main result.

Theorem 1.6. (Homogenization in the limit case)

Assume (H0) and $(\mathbf{H 1})$ and let $\alpha=2$. Let $G$ be defined in (1.12). Then, $U^{\varepsilon}$ converges, locally uniformly on $[0, \infty) \times \mathbb{R}$ and as $\varepsilon \rightarrow 0$, to the unique viscosity solution $\tilde{u}^{0}$ of

$$
\begin{cases}\tilde{u}_{t}^{0}=b\left(\tilde{u}_{x}^{0}\right)\left(\tilde{u}_{x}^{0}\right)^{3}+G\left(\tilde{u}_{x}^{0}\right) \tilde{u}_{x x}^{0} & \text { for }(t, x) \in(0, \infty) \times \mathbb{R} \\ \tilde{u}^{0}(0, x)=u_{0}(x) & \text { for } x \in \mathbb{R},\end{cases}
$$

where $b \in C^{0}(0, \infty)$ satisfies the following explicit formula:

$$
b(p):=\frac{\int_{0}^{1} \frac{\tilde{K}(z, p)}{K(z, p)} h_{z z z}(z, p) h_{z}(z, p) d z}{\int_{0}^{1} \frac{h_{z}^{2}(z, p)}{K(z, p)} d z}
$$


where $h$ is the hull function from Proposition (1.3) item (a) and

$$
\tilde{K}(z, p):=\sum_{1 \leq|i| \leq m} \frac{i^{3}}{6} F_{i}\left(\left[\frac{j^{2}}{2} p^{2} h_{z z}(z, p)\right]_{j}^{m}, h(z, p)\right) .
$$

Assume in particular that $m=2, F\left(V, V_{0}\right)=\sum_{1 \leq|i| \leq 2} l_{i} V_{i}+F_{0}\left(V_{0}\right)$ for a given non-constant 1periodic potential $F_{0} \in C^{2}(\mathbb{R})$ with zero-mean value, and given coefficients such that

$$
\left\{\begin{array}{l}
\min _{1 \leq|i| \leq 2} l_{i} \geq 0 \text { and } \max _{1 \leq|i| \leq 2} l_{i}>0, \\
l_{1}+2 l_{2}=l_{-1}+2 l_{-2} \text { and } l_{1}-l_{-1}+8\left(l_{2}-l_{-2}\right) \neq 0 .
\end{array}\right.
$$

Then $b(p) \neq 0$ for all $p>0$.

A simple example for which the homogenized limit is different is the following variant of the FK model:

$$
\frac{d U_{i}}{d \tau}=2\left(U_{i+1}-U_{i}\right)+\left(U_{i-2}-U_{i}\right)+\varepsilon^{2(\alpha-1)} \sin \left(2 \pi U_{i}\right) .
$$

To show this theorem, we use an ansatz of the same form than in (1.8) with the same hull function $h$, but with a new corrector defined in Proposition A.1.

Let us finally remark that we are studying the homogenization of equations with periodic terms in $u / \varepsilon$, for which only few results exist. In this direction, let us mention the work of Boccardo, Murat [3] about the homogenization of elliptic equations, and the two recent works of Barles [1] and Imbert, Monneau [10].

\section{Organization of the paper}

The paper is organized as follows. Let us emphasize that the proofs of Theorem 1.1 and the convergence result in 1.6 are similar after having computed the ansatz and solved the cell equations. For the sake of simplicity, the full text is devoted to Theorem 1.1 and Appendices A and B to Theorem 1.6. More precisely, Section 2 recalls some basic facts concerning viscosity solutions. The anzatz for Theorem 1.1 is computed in Section 3. Section 4 proves the convergence result of Theorem 1.1. The proofs of the existence and properties of the hull function, the corrector and the diffusion coefficient for Theorem 1.1 are postponed in Section 5. Appendix A gives the new computations for the ansatz and the cell equation for Theorem 1.6. The detailled verification of the rest of the proof of the convergence result is left to the reader. Appendix B proves that $b(p) \neq 0$ under (1.18).

\section{Notations}

Let us introduce the notations that are used throughout.

Integer parts. The floor and the ceiling integer part of a real number $a$ are denoted by $\lfloor a\rfloor$ and $\lceil a\rceil$, respectively.

Cylinders of $(0, \infty) \times \mathbb{R}$. For $\left(t_{0}, x_{0}\right) \in(0, \infty) \times \mathbb{R}$ and $r, R>0$, define $Q_{r, R}\left(t_{0}, x_{0}\right):=\left(t_{0}-r, t_{0}+\right.$ $r) \times\left(x_{0}-R, x_{0}+R\right)$. For the sake of simplicity, $Q_{r, R}\left(t_{0}, x_{0}\right)$ will be sometimes denoted $Q_{r, R}$.

Semi-continuous envelopes. Let $u:[0, \infty) \times \mathbb{R} \rightarrow \mathbb{R}$ be a locally bounded function. We let $u^{*}:[0, \infty) \times \mathbb{R} \rightarrow \mathbb{R}$ denote the smallest upper semi-continuous (u.s.c. for short) function above $u$. The largest lower semi-continuous (l.s.c. for short) function below $u$ is denoted $u_{*}$. 
Relaxed semi-limits. Let $u^{\varepsilon}:[0, \infty) \times \mathbb{R} \rightarrow \mathbb{R}$ be indexed by $\varepsilon>0$. Assume that the family $\left\{u^{\varepsilon}: \varepsilon>0\right\}$ is uniformly locally bounded. The upper relaxed semi-limit of $u^{\varepsilon}$ as $\varepsilon \rightarrow 0$ is the function $\limsup _{\varepsilon \rightarrow 0} u^{\varepsilon}:[0, \infty) \times \mathbb{R} \rightarrow \mathbb{R}$ defined by

$$
\limsup _{\varepsilon \rightarrow 0}^{*} u^{\varepsilon}(t, x):=\limsup _{(\tau, y) \rightarrow(t, x), \varepsilon \rightarrow 0} u^{\varepsilon}(\tau, y) .
$$

The lower relaxed semi-limit is defined by: $\liminf _{\varepsilon \rightarrow 0} u^{\varepsilon}:=-\limsup _{\varepsilon \rightarrow 0}^{*}\left(-u^{\varepsilon}\right)$.

\section{On viscosity solutions}

In this section, we list some basic facts concerning viscosity solutions that are needed throughout. We skip almost all of the proofs, since they are either well-known or straightforward adaptions of classical ones. For a survey on the classical viscosity solutions theory, we refer the reader to the book of Barles [2] and to the user's guide of Crandall, Ishii and Lions [5].

Moreover, let us stress the fact that for $\varepsilon=1$ our system is a special case of the one sudied by Forcadel, Imbert and Monneau, therefore as more related reference one can see Section 2 in [8].

We need to introduce the concept of viscosity solutions because in order to study the system of ODEs (1.1) we embed it into a single PDE. Indeed, by considering $\mathcal{U}(\tau, y):=U_{\lfloor y\rfloor}(\tau)$ we are led to the following "finite-difference like" PDE

$$
\begin{cases}\mathcal{U}_{\tau}(\tau, y)=\varepsilon^{2(\alpha-1)} F\left(\frac{\left[\mathcal{U}\left(\tau, y+j \varepsilon^{\alpha}\right)-\mathcal{U}(\tau, y)\right]_{j}^{m}}{\varepsilon^{2(\alpha-1)}}, \mathcal{U}(\tau, y)\right) & \text { for }(\tau, y) \in(0, \infty) \times \mathbb{R} \\ \mathcal{U}(0, y)=\mathcal{U}_{0}(y) & \text { for } y \in \mathbb{R},\end{cases}
$$

where here $\mathcal{U}_{0}(y):=\frac{1}{\varepsilon} u_{0}\left(\lfloor y\rfloor \varepsilon^{\alpha}\right)$. Note that, because all the results we are going to give are valid for each $\alpha$ and $\varepsilon$ fixed, we will not stress the dependence of the functions $\mathcal{U}$ and $\mathcal{U}_{0}$ on them.

Since the initial datum is sublinear by $(\mathbf{H 0})$, it is natural to restrict ourself to the study of sublinear solutions.

Definition 2.1. (Sublinear functions) A function $u:[0, \infty) \times \mathbb{R} \rightarrow \mathbb{R}$ is sublinear iff for all $T>0, \sup _{(\tau, y) \in[0, T] \times \mathbb{R}} \frac{|u(\tau, y)|}{1+|y|}<\infty$.

Let us recall the notion of viscosity solutions.

\section{Definition 2.2. (Viscosity solutions)}

Let $\mathcal{U}:[0, \infty) \times \mathbb{R} \rightarrow \mathbb{R}$ be sublinear and $\mathcal{U}_{0}: \mathbb{R} \rightarrow \mathbb{R}$ be sublinear. We say that:

1. The function $\mathcal{U}$ is a viscosity subsolution (resp. supersolution) of (2.1) on an open set $\Omega \subset$ $(0, \infty) \times \mathbb{R}$ if $\mathcal{U}$ is u.s.c. (resp. l.s.c.) and for any $\left(\tau_{0}, y_{0}\right) \in \Omega$ and any test function $\phi \in C^{1}(\Omega)$ such that $\mathcal{U}-\phi$ attains a strict local maximum (resp. local minimum) at the point $\left(\tau_{0}, y_{0}\right)$, we have

$$
\phi_{\tau}\left(\tau_{0}, y_{0}\right)-\varepsilon^{2(\alpha-1)} F\left(\frac{\left[\mathcal{U}\left(\tau_{0}, y_{0}+j \varepsilon^{\alpha}\right)-\mathcal{U}\left(\tau_{0}, y_{0}\right)\right]_{j}^{m}}{\varepsilon^{2(\alpha-1)}}, \mathcal{U}\left(\tau_{0}, y_{0}\right)\right) \leq 0 \quad(\text { resp. } \geq 0) .
$$


2. The function $\mathcal{U}$ is a viscosity subsolution (resp. supersolution) of $(2.1)$ in $[0, \infty) \times \mathbb{R}$, if it is a viscosity subsolution (resp. supersolution) on $\Omega=(0, \infty) \times \mathbb{R}$ and if moreover it satisfies

$$
\forall y \in \mathbb{R}, \quad \mathcal{U}(0, y) \leq\left(\mathcal{U}_{0}\right)^{*}(y) \quad\left(\text { resp. } \mathcal{U}(0, y) \geq\left(\mathcal{U}_{0}\right)_{*}(y)\right) .
$$

3. The function $\mathcal{U}$ is a viscosity solution of $(2.1)$ if $\mathcal{U}^{*}$ is a subsolution and $\mathcal{U}_{*}$ is a supersolution.

Here is a comparison principle for the Cauchy problem.

\section{Theorem 2.3. (Comparison principle)}

Assume (H1). Let $u$ and $v$ be respectively a sub-and a supersolution of $(2.1)$ in $(0, \infty) \times \mathbb{R}$. Assume moreover that there exists an uniformly continuous function $\mathcal{V}_{0}$ such that $u(0, y) \leq \mathcal{V}_{0}(y) \leq v(0, y)$ for all $y \in \mathbb{R}$. Then, we have $u \leq v$ on all $[0, \infty) \times \mathbb{R}$.

In the sequel, we shall also need a comparison principle on bounded subdomains. Precisely, let us consider cylinders $Q_{r, R}:=Q_{r, R}\left(t_{0}, x_{0}\right)$ and $Q_{r, R+m}:=Q_{r, R+m}\left(t_{0}, x_{0}\right)$ such that $Q_{r, R} \subset(0, \infty) \times \mathbb{R}$. Then, we have the following result.

\section{Theorem 2.4. (Comparison principle on bounded sets)}

Assume (H1). Let $u$ and $v$ be respectively a sub-and a supersolution of (2.1) in $Q_{r, R}$ such that

$$
u \leq v \quad \text { on } \quad \bar{Q}_{r, R+m} \backslash Q_{r, R}
$$

Then we have $u \leq v$ on $Q_{r, R}$.

Remark 2.5. Let us recall that the PDE in (1.5) is obtained from the one in (2.1), after rescaling by (1.4). In particular, Theorem 2.4 remains true for (1.5) by replacing condition (2.2) by " $u \leq v$ on $\bar{Q}_{r, R+\varepsilon^{\alpha} m} \backslash Q_{r, R} "$

Let us now give an existence and uniqueness result.

Theorem 2.6. (Existence and uniqueness)

Assume (H1) and that $\mathcal{U}_{0}$ is non-decreasing and satisfies:

$$
\sup _{y \in \mathbb{R}}\left\{\mathcal{U}_{0}(y+1)-\mathcal{U}_{0}(y)\right\}<\infty .
$$

Then, there exists a unique viscosity solution of (2.1).

Proof of existence. To get the existence by the classical Perron's method we need to construct barriers. Because of (2.3), the monotonicity of $\mathcal{U}_{0}$ and (H1) (i)-(iii), we see that there exists a constant $C>0$ such that for all $h>0$

$$
u^{+}(\tau, y):=\left(\mathcal{U}_{0}\right)_{*}(y)+C \tau \text { and } u^{-, h}(\tau, y):=\left(\mathcal{U}_{0}\right)^{*}(y-h)-C \tau
$$

satisfy

$$
u^{-, h} \leq u^{+} \text {on }[0, \infty) \times \mathbb{R}
$$

and are respectively a super- and a subsolution of $(2.1)$ on $[0, \infty) \times \mathbb{R}$. By Perron's method, the function

$$
u:=\left(\sup \left\{v: v \leq u^{+}, v \text { is a subsolution }\right\}\right)^{*}
$$


is a solution of $(2.1)$ on $(0, \infty) \times \mathbb{R}$ and satisfy

$$
u^{-, h} \leq u \leq\left(u^{+}\right)^{*} .
$$

Moreover, one have

$$
\begin{array}{r}
\liminf _{h \rightarrow 0} u^{-, h}(\tau, y) \geq\left(\mathcal{U}_{0}\right)_{*}(y)-C \tau, \\
\left(u^{+}\right)^{*}(\tau, y) \leq\left(\mathcal{U}_{0}\right)^{*}(y)+C \tau
\end{array}
$$

(actually, by the monotonicity of $\mathcal{U}_{0}$, the lower semi-limit becomes the limit and the inequalities become equalities). Therefore, letting $h \rightarrow 0$ in (2.4), we obtain

$$
\left(\mathcal{U}_{0}\right)_{*}(y)-C \tau \leq u(\tau, y) \leq\left(\mathcal{U}_{0}\right)^{*}(y)+C \tau,
$$

which implies

$$
u^{*}(0, y) \leq\left(\mathcal{U}_{0}\right)^{*}(y) \text { and } u_{*}(0, y) \geq\left(\mathcal{U}_{0}\right)_{*}(y),
$$

thus $u$ is a viscosity solution of $(2.1)$ on $[0, \infty) \times \mathbb{R}$.

Proof of uniqueness. Let $u$ and $v$ be two viscosity solution of $(2.1)$ on $[0, \infty) \times \mathbb{R}$. We want to show the uniqueness in the following sense:

$$
u^{*}=v^{*} \text { and } u_{*}=v_{*} .
$$

For $h>0$, define $u^{h}(\tau, y):=u(\tau, y-h)$. By the invariance of the equation w.r.t. translations in space, $u^{h}$ is a viscosity solution of $(2.1)$ on $[0, \infty) \times \mathbb{R}$. We plan to compare $\left(u^{h}\right)^{*}=\left(u^{*}\right)^{h}$ and $v_{*}$ by using the comparison principle. To do so, we have to find a good uniformly continuous function $\mathcal{V}_{0}$ between the respective initial data (see Theorem 2.3). Define

$$
\mathcal{V}_{0}(y):=\frac{1}{h} \int_{y-h}^{y} \mathcal{U}_{0}(z) d z
$$

(notice that $\mathcal{U}_{0}$ is measurable as a monotone function). By the monotonicity of $\mathcal{U}_{0}$, it is clear that for all $y_{1}<y_{2}<y_{3},\left(\mathcal{U}_{0}\right)^{*}\left(y_{1}\right) \leq \mathcal{U}_{0}\left(y_{2}\right) \leq\left(\mathcal{U}_{0}\right)_{*}\left(y_{3}\right)$. Hence, we have

$$
\mathcal{V}_{0}(y) \leq \frac{1}{h} \int_{y-h}^{y}\left(\mathcal{U}_{0}\right)_{*}(y) d z=\left(\mathcal{U}_{0}\right)_{*}(y),
$$

for all $y \in \mathbb{R}$. The same way, one prove that

$$
\left(\left(\mathcal{U}_{0}\right)^{*}\right)^{h}(y)=\left(\mathcal{U}_{0}\right)^{*}(y-h) \leq \mathcal{V}_{0}(y)
$$

and conclude that

$$
\left(\left(\mathcal{U}_{0}\right)^{*}\right)^{h} \leq \mathcal{V}_{0} \leq\left(\mathcal{U}_{0}\right)_{*} \quad \text { on } \mathbb{R} .
$$

Moreover, easy computations show that

$$
\left(\mathcal{V}_{0}\right)_{y}(y)=\frac{\mathcal{U}_{0}(y)-\mathcal{U}_{0}(y-h)}{h} \quad \text { for a.e. } y \in \mathbb{R},
$$


which shows under $(2.3)$ and the monotonicity of $\mathcal{U}_{0}$ that $\mathcal{V}_{0}$ is Lipschitz-continuous on $\mathbb{R}$. Since, by the monotonocity of the initial data, we have

$$
\left(u^{h}\right)^{*}(0, y)=\left(u^{*}\right)^{h}(0, y) \leq\left(\left(\mathcal{U}_{0}\right)^{*}\right)^{h}(y)=\left(\mathcal{U}_{0}\right)^{*}(y-h) \leq\left(\mathcal{U}_{0}\right)_{*}(y) \leq v_{*}(0, y) \quad \forall y \in \mathbb{R},
$$

we deduce that $\left(u^{h}\right)^{*}(0, y) \leq \mathcal{V}_{0}(y) \leq v_{*}(0, y)$ and the comparison principle (Theorem 2.3) applies to get

$$
\left(u^{h}\right)^{*} \leq v_{*} \text { on }[0, \infty) \times \mathbb{R} .
$$

Therefore, letting $h \rightarrow 0$, we obtain

$$
u_{*} \leq \liminf _{h \rightarrow 0} u^{h} \leq \liminf _{h \rightarrow 0}\left(u^{h}\right)^{*} \leq v_{*} \text { on }[0, \infty) \times \mathbb{R} .
$$

Similarly, we can exchange the role of $u$ and $v$ from the beginning and obtain

$$
v_{*} \leq u_{*} \text { on }[0, \infty) \times \mathbb{R} .
$$

We thus have proved the equality for the lower enveloppes: $v_{*}=u_{*}$ on $[0, \infty) \times \mathbb{R}$. The proof of the equality for the upper enveloppes being completely similar will be omitted.

Remark 2.7. Actually, we have more information than (2.7); indeed, one have:

$$
v_{*} \geq \liminf _{h \rightarrow 0}\left(u^{h}\right)^{*}=\liminf _{h \rightarrow 0}\left(u^{*}\right)^{h} \geq\left(u^{*}\right)_{*} .
$$

Taking $v=u$, one conclude that $\left(u^{*}\right)_{*}=u_{*}$ and similarly that $\left(u_{*}\right)^{*}=u^{*}$.

To prove the homogeneization result (Theorem 1.1) we will consider the relaxed semilimits of the sequence of solutions $u^{\varepsilon}$ and we will need to prove that those are solutions of the limit equation in the viscosity sense. For the sake of completeness let us recall the definition of viscosity solution in this case and the comparison result we will need in the proof (the proof of the comparison principle for the limit equation being completely classical can be found in [5, Theorem 8.2]).

\section{Definition 2.8. (Viscosity solution: second order)}

Let $u:[0, \infty) \times \mathbb{R} \rightarrow \mathbb{R}$ be sublinear and $u_{0}: \mathbb{R} \rightarrow \mathbb{R}$ be sublinear and continuous. We say that:

1. The function $u$ is a viscosity subsolution (resp. supersolution) to (1.7) in $[0, \infty) \times \mathbb{R}$ if $u$ is u.s.c. (resp. l.s.c.) and for any $\left(t_{0}, x_{0}\right) \in(0, \infty) \times \mathbb{R}$ and any test function $\phi \in C^{\infty}((0, \infty) \times \mathbb{R})$ such that $u-\phi$ attains a strict local maximum (resp. local minimum) at the point $\left(t_{0}, x_{0}\right)$, we have

$$
\phi_{t}\left(t_{0}, x_{0}\right)-G\left(\phi_{x}\left(t_{0}, x_{0}\right)\right) \phi_{x x}\left(t_{0}, x_{0}\right) \leq 0 \quad(\text { resp. } \geq 0),
$$

and if at time zero we have: $\forall x \in \mathbb{R}, u(0, x) \leq u_{0}(x)$ (resp. $u(0, x) \geq u_{0}(x)$ ).

2. The function $u$ is a continuous viscosity solution of (1.7) if it is both viscosity sub- and supersolution.

Theorem 2.9. (Comparison principle) Assume (H0) and (1.6). Let $u$ and $v$ be a viscosity sub- and supersolution of $(1.7)$ in $[0, \infty) \times \mathbb{R}$, respectively. Then $u(t, x) \leq v(t, x)$ for all $(t, x) \in$ $[0, \infty) \times \mathbb{R}$. 


\section{The ansatz}

In order to prove Theorem 1.1, we try to guess the behavior of $U^{\varepsilon}$ around a fixed point $\left(t_{0}, x_{0}\right)$. Our ansatz has been described in (1.8). To verify this thesis, we first have to answer to the following questions:

Question 1. Does this ansatz almost satisfy the PDE in (1.5) around $\left(t_{0}, x_{0}\right)$ ?

Question 2. Does this ansatz converge toward $u^{0}$ as $\varepsilon$ goes to zero, around $\left(t_{0}, x_{0}\right)$ ?

This section is devoted to the verification of these points. Let us first enumerate some required properties on $h$ and $v$ that easily follow from the fact that $h$ (resp. $v$ ) is regular and linear plus periodic (resp. periodic).

\section{Lemma 3.1. (Hull function and corrector properties)}

Assume (H1). For each $\delta>0$, the hull function satisfies:

(a) $C_{0}(\delta):=\max _{\mathbb{R} \times\left[\delta, \frac{1}{\delta}\right]}|h(z, p)-z|<\infty$.

(b) $C_{1}(\delta):=\left\|h_{z}\right\|_{C_{b}^{2}\left(\mathbb{R} \times\left[\delta, \frac{1}{\delta}\right]\right)}+\left\|h_{p}\right\|_{C_{b}^{2}\left(\mathbb{R} \times\left[\delta, \frac{1}{\delta}\right]\right)}<\infty$.

(c) $m(\delta):=\min _{\mathbb{R} \times\left[\delta, \frac{1}{\delta}\right]} h_{z}>0$.

The functions $A$ and $K$ defined in (1.10) satisfy:

(d) $A$ and $K$ are bounded and uniformly continuous on $\mathbb{R} \times\left[\delta, \frac{1}{\delta}\right]$.

Moreover, all corrector (associated to fixed $p_{0}>0$ and $M_{0} \in \mathbb{R}$ ) satisfies

(e) $C_{2}:=\|v\|_{C_{b}^{2}(\mathbb{R})}<\infty$ and $v_{z z}$ is uniformly continuous on $\mathbb{R}$.

\subsection{Verification of the ansatz}

In both lemmata above, we replace $u^{0}$ in (1.8) by general test functions $\phi$ (see (3.3)). This test function will be used in Definition 2.8, when we shall prove the convergence of $U^{\varepsilon}$ by the perturbed test function method in the next section.

For the sake of clarity, we introduce the following notation for the PDE operator in (1.5): for all $\varepsilon>0, \phi \in C^{\infty}((0, \infty) \times \mathbb{R})$ and $(t, x) \in(0, \infty) \times \mathbb{R}$, define

$$
\mathcal{L}_{\varepsilon}[\phi](t, x):=\phi_{t}(t, x)-\frac{1}{\varepsilon} F\left(\left[\frac{\phi\left(t, x+j \varepsilon^{\alpha}\right)-\phi(t, x)}{\varepsilon^{2 \alpha-1}}\right]_{j}^{m}, \frac{\phi(t, x)}{\varepsilon}\right) .
$$

With this notation in hand, the PDE in (1.5) can be rewritten as $\mathcal{L}_{\varepsilon}\left[U^{\varepsilon}\right]=0$.

Here is the main result of this section that we will prove below.

Lemma 3.2. (Local error estimate)

(Settings) Assume $(\mathbf{H 1})$ and $\mathbf{( H 2 )}$. Let $\phi \in C^{\infty}((0, \infty) \times \mathbb{R})$ be such that

$$
\phi_{x}\left(t_{0}, x_{0}\right)>0 \text { for some fixed }\left(t_{0}, x_{0}\right) \in(0, \infty) \times \mathbb{R} \text {. }
$$


Let $\lambda$ be defined by Proposition 1.3 item (b) with $\left(p_{0}, M_{0}\right):=\left(\phi_{x}\left(t_{0}, x_{0}\right), \phi_{x x}\left(t_{0}, x_{0}\right)\right)$, and let $v$ be an associated corrector. Let $h$ be the hull function of Proposition 1.3 and for $\varepsilon>0$, define

$$
\phi^{\varepsilon}(t, x):=\varepsilon h\left(\frac{\tilde{\phi}^{\varepsilon}(t, x)}{\varepsilon}, \phi_{x}(t, x)\right) \quad \text { with } \quad \tilde{\phi}^{\varepsilon}(t, x):=\phi(t, x)+\varepsilon^{2} v\left(\frac{\phi(t, x)}{\varepsilon}\right) .
$$

(Result) Then, $\phi^{\varepsilon}(t, x)$ and $\mathcal{L}_{\varepsilon}\left[\phi^{\varepsilon}\right](t, x)$ are well-defined for $(t, x, \varepsilon)$ sufficiently close to $\left(t_{0}, x_{0}, 0\right)$. Moreover, we have

$$
\mathcal{L}_{\varepsilon}\left[\phi^{\varepsilon}\right](t, x)=\left(\phi_{t}(t, x)-\lambda+\mathcal{E}(t, x, \varepsilon)\right) h_{\tilde{z}} \quad \text { with } \lim _{(t, x, \varepsilon) \rightarrow\left(t_{0}, x_{0}, 0\right)}|\mathcal{E}(t, x, \varepsilon)|=0,
$$

where we set $h_{\tilde{z}}=h_{\tilde{z}}\left(\tilde{z}, \phi_{x}(t, x)\right)$ with $\tilde{z}=\frac{\tilde{\phi}^{\varepsilon}(t, x)}{\varepsilon}$.

Lemma 3.3. (Convergence toward $u^{0}$ )

Assume (H1) and (H2) and let $\phi^{\varepsilon}$ be defined by (3.3). Then, there exists $r>0$ such that for all $\varepsilon>0, \phi^{\varepsilon}$ is well-defined on $Q_{r, r}=Q_{r, r}\left(t_{0}, x_{0}\right)$ and converges toward $\phi$, as $\varepsilon \rightarrow 0$, uniformly on $Q_{r, r}$.

Proof of Lemma 3.3. The thesis easily follows from Lemma 3.1 items (a) and (e).

Remark 3.4. Taking $\phi=u^{0}$, our ansatz in (1.8) writes $U^{\varepsilon} \approx\left(u^{0}\right)^{\varepsilon}$ around $\left(t_{0}, x_{0}\right)$. These lemmata then mean formally that we indeed have $\mathcal{L}_{\varepsilon}\left[\left(u^{0}\right)^{\varepsilon}\right] \approx 0$ and $\left(u^{0}\right)^{\varepsilon} \approx u^{0}$ around $\left(t_{0}, x_{0}\right)$.

Proof of Lemma 3.2. Let us first remark that without loss of generality we can replace the assumption $\phi \in C^{\infty}((0, \infty) \times \mathbb{R})$ by the following:

$$
\left\{\begin{array}{l}
\phi \text { is Lipschitz-continuous on }(0, \infty) \times \mathbb{R} \text { with } \delta \leq \phi_{x} \leq \frac{1}{\delta} \text { for some } \delta>0 \\
\left|\phi_{x}\right|+\left|\phi_{x x}\right|+\left|\phi_{x x x}\right| \leq C_{\phi} \text { on }(0, \infty) \times \mathbb{R} \text { for some constant } C_{\phi} . \\
\left|\phi_{t}\right|+\left|\phi_{x t}\right| \leq C_{\phi}^{\prime} \text { on }(0, \infty) \times \mathbb{R} \text { for some constant } C_{\phi}^{\prime} .
\end{array}\right.
$$

Indeed, it is clear from (3.2) that $\delta \leq \phi_{x} \leq \frac{1}{\delta}$ on $Q_{r, 2 r}=Q_{r, 2 r}\left(t_{0}, x_{0}\right)$, for some $\delta>0$ and $r>0$. It follows that $\phi^{\varepsilon}$ is well-defined on $Q_{r, 2 r}$, since the second argument of $h$ in (3.3) is positive. By (3.1), it is also obvious that for all $\varepsilon \leq\left(\frac{r}{m}\right)^{\frac{1}{\alpha}}, \mathcal{L}_{\varepsilon}\left[\phi^{\varepsilon}\right]$ is well-defined on $Q_{r, r}$ and only depends on the values of $\phi$ on $Q_{r, r+\varepsilon^{\alpha} m} \subseteq Q_{r, 2 r}$. In particular, it is easy to modify $\phi$ outside $Q_{r, 2 r}$ in order to verify (3.5), without changing the value of $\mathcal{L}_{\varepsilon}\left[\phi^{\varepsilon}\right](t, x)$ for $(t, x, \varepsilon)$ close to $\left(t_{0}, x_{0}, 0\right)$.

Therefore we will prove (3.4) under the additional assumption (3.5). Our strategy is the following. First, we develop $\mathcal{L}_{\varepsilon}\left[\phi^{\varepsilon}\right]$ w.r.t. $\varepsilon$ by using Taylor's formula. This will give an expression of the form:

$$
\mathcal{L}_{\varepsilon}\left[\phi^{\varepsilon}\right](t, x)=\frac{1}{\varepsilon}(\ldots)+\varepsilon^{0}(\ldots)+o(1),
$$

where $o(1)$ denotes an error that vanishes as $\varepsilon \rightarrow 0$. Second, we use the cell equations (1.9) and (1.11), in order to vanish respectively the terms of order $\frac{1}{\varepsilon}$ and $\varepsilon^{0}$.

The rest of the proof is organized in several steps. In the first step, we introduce some notations that shall be used throughout and the successive Taylor's expansions used to get (3.6) are detailled in the other steps. 
Step 1: slow and fast variables. Define

$$
\tilde{z}:=\frac{\tilde{\phi}^{\varepsilon}(t, x)}{\varepsilon}, \quad z:=\frac{\phi(t, x)}{\varepsilon} \text { and } \quad p:=\phi_{x}(t, x) .
$$

The functions $\phi^{\varepsilon}$ and $\tilde{\phi}^{\varepsilon}$ then write

$$
\phi^{\varepsilon}(t, x)=\varepsilon h(\tilde{z}, p) \quad \text { and } \quad \tilde{\phi}^{\varepsilon}(t, x)=\phi(t, x)+\varepsilon^{2} v(z) .
$$

In order to simplify the notations, we will not precise if not necessary the variables of $\phi, \tilde{\phi}^{\varepsilon}, \phi^{\varepsilon}, h$ and $v$. Without any more precision, the reader will have to understood that these functions and their derivatives are expressed at the variables as in (3.7).

Step 2: first equation in $\phi^{\varepsilon}$. We have

$$
\phi_{t}^{\varepsilon}=h_{\tilde{z}} \tilde{\phi}_{t}^{\varepsilon}+\varepsilon h_{p} \phi_{x t}=h_{\tilde{z}} \phi_{t}+\varepsilon\left(h_{\tilde{z}} v_{z} \phi_{t}+h_{p} \phi_{x t}\right) .
$$

Consequently, we get:

$$
\mathcal{L}_{\varepsilon}\left[\phi^{\varepsilon}\right]=h_{\tilde{z}} \phi_{t}+\varepsilon\left(h_{\tilde{z}} v_{z} \phi_{t}+h_{p} \phi_{x t}\right)-\frac{1}{\varepsilon} F\left(\frac{1}{\varepsilon^{2 \alpha-2}} V, h\right),
$$

where we set:

$$
V:=\left[V_{j}\right]_{j}^{m}=\left[h\left(\frac{\tilde{\phi}^{\varepsilon}\left(t, x+j \varepsilon^{\alpha}\right)}{\varepsilon}, \phi_{x}\left(t, x+j \varepsilon^{\alpha}\right)\right)-h(\tilde{z}, p)\right]_{j}^{m} .
$$

Step 3: expansion of $V$. In this step, we develop the first argument $\frac{1}{\varepsilon^{2 \alpha-2}} V$ of $F$. Let us begin by developing $\phi_{x}$ and $\tilde{\phi}^{\varepsilon}$ around $(t, x)$.

We claim that for all $1 \leq|j| \leq m,(t, x) \in(0, \infty) \times \mathbb{R}$ and $\varepsilon>0$ we have

$$
\left\{\begin{array}{l}
\tilde{\phi}^{\varepsilon}\left(t, x+j \varepsilon^{\alpha}\right)=\tilde{\phi}^{\varepsilon}+j \varepsilon^{\alpha} \tilde{\phi}_{x}^{\varepsilon}+\frac{j^{2}}{2} \varepsilon^{2 \alpha} \tilde{\phi}_{x x}^{\varepsilon}+\tilde{R}(t, x, j, \varepsilon), \\
\text { with } \frac{|\tilde{R}(t, x, j, \varepsilon)|}{\varepsilon^{2 \alpha}} \text { as } \varepsilon \rightarrow 0 \text { uniformly in }(t, x, j) .
\end{array}\right.
$$

By the classical Taylor's formula, we have:

$$
|\tilde{R}(t, x, j, \varepsilon)| \leq j^{2} \varepsilon^{2 \alpha} \frac{\omega_{\varepsilon}\left(|j| \varepsilon^{\alpha}\right)}{2},
$$

where $\omega_{\varepsilon}(\cdot)$ is the modulus of continuity in $x$ of $\tilde{\phi}_{x x}^{\varepsilon}=\phi_{x x}+v_{z z} \phi_{x}^{2}+\varepsilon v_{z} \phi_{x x}$ (by Definition (3.3)). Let us estimate the modulus of continuity of these three terms. The most difficult is the middle term $v_{z z} \phi_{x}^{2}$. Let $\left(x, x^{\prime}\right) \in \mathbb{R}$ be such that $\left|x-x^{\prime}\right| \leq \beta$. We have

$$
\begin{aligned}
I:=\mid v_{z z}\left(\frac{\phi(t, x)}{\varepsilon}\right) \phi_{x}^{2}(t, x)-v_{z z}\left(\frac{\phi\left(t, x^{\prime}\right)}{\varepsilon}\right) & \phi_{x}^{2}\left(t, x^{\prime}\right) \mid \\
& \leq C_{2} C_{\phi} \beta+C_{\phi}^{2}\left|v_{z z}\left(\frac{\phi(t, x)}{\varepsilon}\right)-v_{z z}\left(\frac{\phi\left(t, x^{\prime}\right)}{\varepsilon}\right)\right|,
\end{aligned}
$$


where $C_{2}$ is the bound on $v_{z z}$ in Lemma 3.1 (e) and $C_{\phi}$ is the bound in (3.5). We have

$$
\left|v_{z z}\left(\frac{\phi(t, x)}{\varepsilon}\right)-v_{z z}\left(\frac{\phi\left(t, x^{\prime}\right)}{\varepsilon}\right)\right| \leq \omega_{v}\left(\frac{\phi(t, x)-\phi\left(t, x^{\prime}\right)}{\varepsilon}\right) \leq \omega_{v}\left(\frac{C_{\phi}}{\varepsilon} \beta\right),
$$

where $\omega_{v}(\cdot)$ is the modulus of continuity of $v_{z z}$ on $\mathbb{R}$ from Lemma 3.1 (e). It follows that $I \leq$ $C_{2} C_{\phi} \beta+C_{\phi}^{2} \omega_{v}\left(\frac{C_{\phi}}{\varepsilon} \beta\right)$, which proves that the modulus of continuity $\omega(\cdot)$ of $v_{z z} \phi_{x}^{2}$ satisfies

$$
\omega(\beta) \leq C_{2} C_{\phi} \beta+C_{\phi}^{2} \omega_{v}\left(\frac{C_{\phi}}{\varepsilon} \beta\right) \quad \text { for all } \beta>0 .
$$

Since (3.5) and Lemma 3.1 (e) imply that $\phi_{x x}+\varepsilon v_{z} \phi_{x x}$ is Lipschitz-continuous in $x$ with a Lipschitzconstant $L_{\phi}$ independent on small $\varepsilon$, we get

$$
\omega_{\varepsilon}(\beta) \leq L_{\phi} \beta+C_{2} C_{\phi} \beta+C_{\phi}^{2} \omega_{v}\left(\frac{C_{\phi}}{\varepsilon} \beta\right) .
$$

Thus (3.11) and $\alpha>0$ imply $\tilde{R}(t, x, j, \varepsilon)=o\left(\varepsilon^{2 \alpha}\right)$ and the proof of (3.10) is complete.

Recall that $\tilde{\phi}_{x}^{\varepsilon}=p+\varepsilon v_{z} p$ and $\tilde{\phi}_{x x}^{\varepsilon}=\phi_{x x}+v_{z z} p^{2}+\varepsilon v_{z} \phi_{x x}$. Dividing (3.10) by $\varepsilon$, we get

$$
\begin{aligned}
\frac{\tilde{\phi}^{\varepsilon}\left(t, x+j \varepsilon^{\alpha}\right)}{\varepsilon} & =\frac{\tilde{\phi}^{\varepsilon}}{\varepsilon}+j \varepsilon^{\alpha-1} \tilde{\phi}_{x}^{\varepsilon}+\frac{j^{2}}{2} \varepsilon^{2 \alpha-1} \tilde{\phi}_{x x}^{\varepsilon}+o\left(\varepsilon^{2 \alpha-1}\right) \\
& =\tilde{z}+j \varepsilon^{\alpha-1} p+j \varepsilon^{\alpha} v_{z} p+\frac{j^{2}}{2} \varepsilon^{2 \alpha-1}\left(\phi_{x x}+v_{z z} p^{2}\right)+o\left(\varepsilon^{2 \alpha-1}\right) \\
& :=\tilde{z}+\mathcal{E}_{1} .
\end{aligned}
$$

Next, from (3.5) we deduce that

$$
\phi_{x}\left(t, x+j \varepsilon^{\alpha}\right)=p+j \varepsilon^{\alpha} \phi_{x x}+o\left(\varepsilon^{\alpha}\right):=p+\mathcal{E}_{2} .
$$

We can now develop the term $h(\ldots, \ldots)$ in $(3.9)$ around $(\tilde{z}, p)$. We get the following expansion

$$
\begin{array}{r}
h\left(\frac{\tilde{\phi}^{\varepsilon}\left(t, x+j \varepsilon^{\alpha}\right)}{\varepsilon}, \phi_{x}\left(t, x+j \varepsilon^{\alpha}\right)\right)-h(\tilde{z}, p)=h\left(\tilde{z}+\mathcal{E}_{1}, p+\mathcal{E}_{2}\right)-h(\tilde{z}, p) \\
=h_{\tilde{z}} \mathcal{E}_{1}+h_{p} \mathcal{E}_{2}+\frac{h_{\tilde{z} \tilde{z}}}{2} \mathcal{E}_{1}^{2}+\frac{h_{p p}}{2} \mathcal{E}_{2}^{2}+h_{\tilde{z} p} \mathcal{E}_{1} \mathcal{E}_{2}+R
\end{array}
$$

for some rest $R=R(t, x, j, \varepsilon)$. Since $h$ is $C^{3}$, Taylor's Young formula implies that

$$
|R| \leq\left(\left|\mathcal{E}_{1}\right|+\left|\mathcal{E}_{2}\right|\right)^{3} \frac{1}{3 !} \sup _{\mathcal{D}}\left|D^{3} h\right|
$$

where $\mathcal{D}$ is the segment of extremities $(\tilde{z}, p)$ and $\left(\frac{\tilde{\phi}^{\varepsilon}\left(t, x+j \varepsilon^{\alpha}\right)}{\varepsilon}, \phi_{x}\left(t, x+j \varepsilon^{\alpha}\right)\right)$. But, both these points belong to $\mathbb{R} \times\left[\delta, \frac{1}{\delta}\right]$ by (3.5). Hence, Lemma 3.1 implies that

$$
|R| \leq\left(\left|\mathcal{E}_{1}\right|+\left|\mathcal{E}_{2}\right|\right)^{3} \frac{C_{1}(\delta)}{3 !} .
$$


Since the term of lowest order in $\left|\mathcal{E}_{1}\right|+\left|\mathcal{E}_{2}\right|$ is $j \varepsilon^{\alpha-1} p$, the better estimate we can have for $R$ is $|R| \leq C \varepsilon^{3(\alpha-1)}$ (the constant $C$ does not depend on $\varepsilon$ sufficiently small, $(t, x) \in(0, \infty) \times \mathbb{R}$ and $1 \leq|j| \leq m$, since the other terms of $\left|\mathcal{E}_{1}\right|+\left|\mathcal{E}_{2}\right|$ are controlled by (3.5) and Lemma 3.1). Since by (H2) $\alpha$ is assumed greater than 2 , we get a fortiori

$$
R=o\left(\varepsilon^{2 \alpha-1}\right) .
$$

Let us stress the fact that this is the only point in which (H2) is used. All the other estimates in the paper are valid for $\alpha>1$.

Let us now develop all the terms in (3.14). In order to control all the negligible terms, we use (3.5) and Lemma 3.1. We get:

$$
\begin{aligned}
& h_{\tilde{z}} \mathcal{E}_{1}=j h_{\tilde{z}}\left(\varepsilon^{\alpha-1} p+\varepsilon^{\alpha} v_{z} p\right)+\varepsilon^{2 \alpha-1} \frac{j^{2}}{2} h_{\tilde{z}}\left(\phi_{x x}+v_{z z} p^{2}\right)+o\left(\varepsilon^{2 \alpha-1}\right) . \\
& h_{p} \mathcal{E}_{2}=j \varepsilon^{\alpha} h_{p} \phi_{x x}+o\left(\varepsilon^{\alpha}\right) \text {. } \\
& \frac{h_{\tilde{z} \tilde{z}}}{2} \mathcal{E}_{1}^{2}=\varepsilon^{2 \alpha-2} \frac{j^{2}}{2} h_{\tilde{z} \tilde{z}} p^{2}+\varepsilon^{2 \alpha-1} j^{2} h_{\tilde{z} \tilde{z}} v_{z} p^{2}+o\left(\varepsilon^{2 \alpha-1}\right) \text {. } \\
& \frac{h_{p p}}{2} \mathcal{E}_{2}^{2}=o\left(\varepsilon^{2 \alpha-1}\right) \text {. } \\
& h_{\tilde{z} p} \mathcal{E}_{1} \mathcal{E}_{1}=\varepsilon^{2 \alpha-1} j^{2} h_{\tilde{z} p} p \phi_{x x}+o\left(\varepsilon^{2 \alpha-1}\right) \text {. }
\end{aligned}
$$

Plugging this into (3.14), we get the following expansion:

$$
\begin{aligned}
& h\left(\frac{\tilde{\phi}^{\varepsilon}\left(t, x+j \varepsilon^{\alpha}\right)}{\varepsilon}, \phi_{x}\left(t, x+j \varepsilon^{\alpha}\right)\right)-h(\tilde{z}, p)= \\
& =j\left\{h_{\tilde{z}}\left(\varepsilon^{\alpha-1} p+\varepsilon^{\alpha} v_{z} p\right)+\varepsilon^{\alpha} h_{p} \phi_{x x}\right\}+\varepsilon^{2 \alpha-2}\left\{\frac{j^{2}}{2} h_{\tilde{z} \tilde{z} p^{2}}\right\} \\
& \quad+\varepsilon^{2 \alpha-1}\left\{\frac{j^{2}}{2}\left(h_{\tilde{z}}\left(\phi_{x x}+v_{z z} p^{2}\right)+2 h_{\tilde{z} \tilde{z}} v_{z} p^{2}+2 h_{\tilde{z} p} p \phi_{x x}\right)\right\}+o\left(\varepsilon^{2 \alpha-1}\right) .
\end{aligned}
$$

Dividing (3.16) by $\varepsilon^{2 \alpha-2}$, we get the following expansion of $V$ in (3.9):

$$
\begin{aligned}
& \frac{1}{\varepsilon^{2 \alpha-2}} V_{j}=\frac{1}{\varepsilon^{2 \alpha-2}}\left\{h\left(\frac{\tilde{\phi}^{\varepsilon}\left(t, x+j \varepsilon^{\alpha}\right)}{\varepsilon}, \phi_{x}\left(t, x+j \varepsilon^{\alpha}\right)\right)-h(\tilde{z}, p)\right\} \\
& =j q+\frac{j^{2}}{2} h_{\tilde{z} \tilde{z}} p^{2}+\varepsilon j^{2} h_{\tilde{z}}\left\{A \phi_{x x}+p^{2}\left(\frac{v_{z z}}{2}+\frac{h_{\tilde{z} \tilde{z}}}{h_{\tilde{z}}} v_{z}\right)\right\}+o(\varepsilon)
\end{aligned}
$$

where $A=A(\tilde{z}, p)$ is defined in (1.10) and $q:=\frac{1}{\varepsilon^{2 \alpha-2}}\left\{h_{\tilde{z}}\left(\varepsilon^{\alpha-1} p+\varepsilon^{\alpha} v_{z} p\right)+\varepsilon^{\alpha} h_{p} \phi_{x x}\right\}$ is a linear displacement.

Step 4: removing linear displacement. This step is crucial, since $q$ contains terms of order $\varepsilon^{-\alpha+1}$ 
and $\varepsilon^{-\alpha+2}$ that can not be controlled. We thus use (H1) (iv) to see from (3.17) that

$$
\begin{aligned}
& F\left(\frac{1}{\varepsilon^{2 \alpha-2}} V, h\right) \\
& =F\left(\left[j q+\frac{j^{2}}{2} h_{\tilde{z} \tilde{z}} p^{2}+\varepsilon j^{2} h_{\tilde{z}}\left\{A \phi_{x x}+p^{2}\left(\frac{v_{z z}}{2}+\frac{h_{\tilde{z} \tilde{z}}}{h_{\tilde{z}}} v_{z}\right)\right\}+o(\varepsilon)\right]_{j}^{m}, h\right) \\
& \quad=F\left(\left[\frac{j^{2}}{2} h_{\tilde{z} \tilde{z}} p^{2}\right]_{j}^{m}+\varepsilon\left[j^{2} h_{\tilde{z}}\left\{A \phi_{x x}+p^{2}\left(\frac{v_{z z}}{2}+\frac{h_{\tilde{z} \tilde{z}}}{h_{\tilde{z}}} v_{z}\right)\right]_{j}^{m}+o(\varepsilon), h\right) .\right.
\end{aligned}
$$

Step 5: expansion of $F$. We develop $F$ around $\left(\left[\frac{j^{2}}{2} h_{\tilde{z} \tilde{z}} p^{2}\right]_{j}^{m}, h\right)$.

We claim that there exists a constant $R_{\phi}$ such that for all $\varepsilon$ small enough and all $(t, x) \in(0, \infty) \times \mathbb{R}$, the first argument of $F$ in the third line of (3.18) is bounded by the constant $R_{\phi}$. To see this, we simply use one more time (3.5) and Lemma 3.1. With these observations in hands, it is clear from Taylor's formula that

$$
\begin{aligned}
F\left(\frac{1}{\varepsilon^{2 \alpha-2}} V, h\right) & =F\left(\left[\frac{j^{2}}{2} h_{\tilde{z} \tilde{z}} p^{2}\right]_{j}^{m}, h\right) \\
& +\varepsilon\left\{\sum_{1 \leq|i| \leq m} i^{2} F_{i}\left(\left[\frac{j^{2}}{2} h_{\tilde{z} \tilde{z}} p^{2}\right]_{j}^{m}, h\right)\right\} h_{\tilde{z}}\left\{A \phi_{x x}+p^{2}\left(\frac{v_{z z}}{2}+\frac{h_{\tilde{z} \tilde{z}}}{h_{\tilde{z}}} v_{z}\right)\right\}+o(\varepsilon),
\end{aligned}
$$

where $o(\varepsilon)$ depends only on $R_{\phi}$, and on the modulus of continuity and the $L^{\infty}$-norms of the firstorder derivatives of $F$ on $\bar{B}_{R_{\phi}} \times \mathbb{R}$. Notice that this modulus is finite, thanks to (H1) (i)-(ii). Dividing this equation by $\varepsilon$, we get:

$$
\begin{aligned}
& \frac{1}{\varepsilon} F\left(\frac{1}{\varepsilon^{2 \alpha-2}} V, h\right)=\frac{1}{\varepsilon} F\left(\left[\frac{j^{2}}{2} h_{\tilde{z} z} p^{2}\right]_{j}^{m}, h\right) \\
& +\left\{\sum_{1 \leq|i| \leq m} i^{2} F_{i}\left(\left[\frac{j^{2}}{2} h_{\tilde{z} \tilde{z}} p^{2}\right]_{j}^{m}, h\right)\right\} h_{\tilde{z}}\left\{A \phi_{x x}+p^{2}\left(\frac{v_{z z}}{2}+\frac{h_{\tilde{z} \tilde{z}}}{h_{\tilde{z}}} v_{z}\right)\right\}+o(1) \\
& =\frac{1}{\varepsilon} F\left(\left[\frac{j^{2}}{2} h_{\tilde{z} \tilde{z}} p^{2}\right]_{j}^{m}, h\right)+h_{\tilde{z}}\left\{K A \phi_{x x}+K p^{2}\left(\frac{v_{z z}}{2}+\frac{h_{\tilde{z} \tilde{z}}}{h_{\tilde{z}}} v_{z}\right)\right\}+o(1),
\end{aligned}
$$

where $K=K(\tilde{z}, p)$ is defined in (1.10).

Step 6: conclusion. By (3.19), equation (3.8) becomes:

$$
\begin{aligned}
\mathcal{L}_{\varepsilon}\left[\phi^{\varepsilon}\right]=h_{\tilde{z}} \phi_{t} & +\varepsilon\left(h_{\tilde{z}} v_{z} \phi_{t}+h_{p} \phi_{x t}\right) \\
& -\frac{1}{\varepsilon} F\left(\left[\frac{j^{2}}{2} h_{\tilde{z} \tilde{z}} p^{2}\right]_{j}^{m}, h\right)-h_{\tilde{z}}\left\{K A \phi_{x x}+K p^{2}\left(\frac{v_{z z}}{2}+\frac{h_{\tilde{z} \tilde{z}}}{h_{\tilde{z}}} v_{z}\right)\right\}+o(1) .
\end{aligned}
$$


Our aim is now to use the cell systems (1.9) and (1.11) to control the terms of order $\frac{1}{\varepsilon}$ and $\varepsilon^{0}$. First, we see from (1.9) that

$$
\frac{1}{\varepsilon} F\left(\left[\frac{j^{2}}{2} h_{\tilde{z} \tilde{z}}\right]_{j}^{m}, h\right)=\frac{1}{\varepsilon} F\left(\left[\frac{j^{2}}{2} h_{\tilde{z} \tilde{z}}(\tilde{z}, p)\right]_{j}^{m}, h(\tilde{z}, p)\right)=0 .
$$

Moreover, by (3.5) and Lemma 3.1, we have

$$
\left|h_{\tilde{z}} v_{z} \phi_{t}+h_{p} \phi_{x t}\right| \leq C_{1}(\delta) C_{\phi}^{\prime}\left(C_{2}+1\right) .
$$

Hence, the $\varepsilon$-term in (3.20) can be included in the $o(1)$. Therefore, we obtain:

$$
\mathcal{L}_{\varepsilon}\left[\phi^{\varepsilon}\right](t, x)=h_{\tilde{z}}(\tilde{z}, p)\left[\phi_{t}(t, x)-\mathcal{G}(z, \tilde{z}, p, M)\right]+o(1),
$$

where we set

$$
\mathcal{G}(z, \tilde{z}, p, M):=K(\tilde{z}, p) A(\tilde{z}, p) M+K(\tilde{z}, p) p^{2}\left(\frac{v_{z z}(z)}{2}+\frac{h_{\tilde{z} \tilde{z}}(\tilde{z}, p)}{h_{\tilde{z}}(\tilde{z}, p)} v_{z}(z)\right)
$$

with $M:=\phi_{x x}(t, x)$. By (1.11), we have $\lambda=\mathcal{G}\left(z, z, p_{0}, M_{0}\right)$ and thus

$$
\mathcal{L}_{\varepsilon}\left[\phi^{\varepsilon}\right](t, x)=h_{\tilde{z}}(\tilde{z}, p)\left(\phi_{t}(t, x)-\lambda+\mathcal{G}\left(z, z, p_{0}, M_{0}\right)-\mathcal{G}(z, \tilde{z}, p, M)\right)+o(1) .
$$

The thesis follows now from the regularity of $\phi$, the estimate (3.5) and the uniform continuity of $\mathcal{G}$ on $\mathbb{R} \times(0, \infty) \times\left[\delta, \frac{1}{\delta}\right] \times\left[-C_{\phi}, C_{\phi}\right]$, thanks to Lemma 3.1 (b)-(e).

\section{Proof of the homogenization result}

Proof of Theorem 1.1. We first notice that for each $\varepsilon>0$ fixed, the general Theorem 2.6 applies and we have the existence and uniqueness of the solution of (1.5). Moreover, Proposition 1.4 states the existence and the regularity of the function $G$ required in (1.6). This regularity allows us to apply the now classical results on viscosity solution to obtain the existence and uniqueness of a viscosity solution of the limit equation (1.7) (See for instance [5]).

We consider now equation (1.5) with the continuous initial datum $u_{0}$, more precisely:

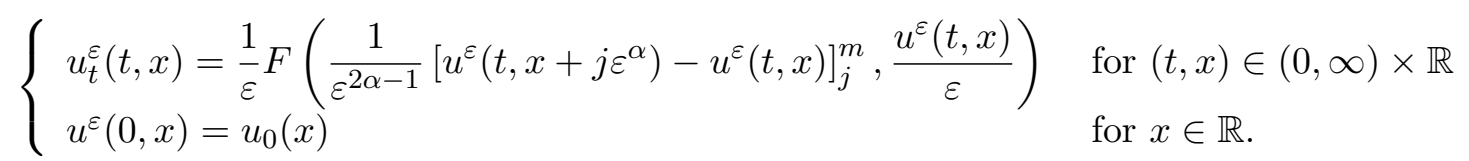

We claim that if we prove that the sequence of solutions $u^{\varepsilon}$ of (4.1) converges to $u^{0}$, as $\varepsilon$ goes to zero, then our convergence result on the sequence $U^{\varepsilon}$ follows (remark that Theorem 2.6 in particular implies the existence and the uniqueness of a continuous viscosity solution $u^{\varepsilon}$ of (4.1)).

Let us justify our claim. By the 1-periodicity of $F$ in (H1) (ii), the PDE in (1.5) is invariant w.r.t. $\varepsilon \times$ integer additions (that is to say, if $u^{\varepsilon}$ is solution of (4.1), then $u+\varepsilon n$ would satisfy the PDE in (1.5) for all $n \in \mathbb{Z})$. Therefore $u^{\varepsilon}(t, x)-\varepsilon\left\lceil\frac{\varepsilon^{\alpha}}{\varepsilon \delta_{0}}\right\rceil$ is a viscosity solution of (4.1) with initial condition $u_{0}(x)-\varepsilon\left\lceil\frac{\varepsilon^{\alpha}}{\varepsilon \delta_{0}}\right\rceil$. Moreover, since at initial time we have

$$
u_{0}(x)-\varepsilon\left\lceil\frac{\varepsilon^{\alpha}}{\varepsilon \delta_{0}}\right\rceil \leq u_{0}\left(\left\lfloor\frac{x}{\varepsilon^{\alpha}}\right\rfloor \varepsilon^{\alpha}\right) \leq u_{0}(x) \quad \forall x \in \mathbb{R},
$$


the function $U^{\varepsilon}$ is a viscosity subsolution (resp. supersolution) of (4.1) with a uniformly continuous initial datum $u_{0}$ (resp. $u_{0}-\varepsilon\left\lceil\frac{\varepsilon^{\alpha}}{\varepsilon \delta_{0}}\right\rceil$ ). Thus the comparison principle (Theorem 2.3) implies that

$$
u^{\varepsilon}(t, x)-\varepsilon\left\lceil\frac{\varepsilon^{\alpha}}{\varepsilon \delta_{0}}\right\rceil \leq U^{\varepsilon}(t, x) \leq u^{\varepsilon}(t, x) \quad \forall(t, x) \in[0, \infty) \times \mathbb{R} .
$$

Hence, if $u^{\varepsilon}$ converges toward $u^{0}$, as $\varepsilon \rightarrow 0$, then $U^{\varepsilon}$ would also converge toward $u^{0}$.

Let us now prove the convergence result for $u^{\varepsilon}$ solution of (4.1). The proof will follow the classical method of building suitable perturbed test functions. Let us denote the relaxed semilimits of the sequence $u^{\varepsilon}$ by:

$$
\bar{u}(t, x)=\limsup _{\varepsilon \rightarrow 0}{ }^{*} u^{\varepsilon}(t, x), \quad \underline{u}(t, x)=\liminf _{\varepsilon \rightarrow 0}{ }_{*} u^{\varepsilon}(t, x) .
$$

Our aim is to prove that $\bar{u}$ and $\underline{u}$ are respectively viscosity sub- and super solution of the limit problem (1.7) in $[0, \infty) \times \mathbb{R}$. Indeed, if this is true by the comparison result for the limit equation (Theorem 2.9), we have $\bar{u}(t, x) \leq \underline{u}(t, x)$ for all $(t, x)$ in $(0, \infty) \times \mathbb{R}$. By construction we have the reverse inequality, thus, as $\varepsilon$ tends to 0 we will have

$$
u^{\varepsilon}(t, x) \rightarrow \bar{u}(t, x)=\underline{u}(t, x):=u^{0}(t, x) \text { uniformly on compact set of }[0, \infty) \times \mathbb{R},
$$

which will be the unique solution of (1.7) and this will give us the thesis.

We will proceed in three steps. First, we will construct $\varepsilon$-uniform barriers on $u^{\varepsilon}$ to be sure that the relaxed semi-limit are well defined. Secondly, we will prove a uniform bound on the gradients of $\bar{u}$ and $\underline{u}$ in order to be allowed to choose $p=\phi_{x}=\bar{u}_{x}>0$ (or $p=\phi_{x}=\underline{u}_{x}>0$ ) in the third step. Finally, we will prove that $\bar{u}$ is a subsolution of (1.7) (the proof of $\underline{u}$ being a supersolution being completely similar will be not detailed).

Step 1. Barriers on $u^{\varepsilon}$. The idea is to construct a sub- and a supersolution of (4.1) not depending on $\varepsilon$, or at least locally bounded uniformly in $\varepsilon$. To do this, we look for semi-solutions in the form of the ansatz (1.8). Precisely, for $(t, x) \in[0, \infty) \times \mathbb{R}$ and $\varepsilon>0$, define

$$
u^{ \pm, \varepsilon}(t, x):=\varepsilon h\left(\frac{u_{0}(x) \pm C t}{\varepsilon},\left(u_{0}\right)_{x}(x)\right) \pm \varepsilon\left\lceil C_{0}\left(\delta_{0}\right)\right\rceil,
$$

where $C$ is a positive constant that will be appropriately chosen later. Let us recall that these functions are well-defined, since $\left(u_{0}\right)_{x} \geq \delta_{0}>0$ on $\mathbb{R}$.

Proof of $u^{+, \varepsilon}$ is a supersolution of (1.5). We begin by verifying the initial condition. By Lemma 3.1 item (a), we have for all $x \in \mathbb{R}$,

$$
u^{+, \varepsilon}(0, x)=\varepsilon h\left(\frac{u_{0}(x)}{\varepsilon},\left(u_{0}\right)_{x}(x)\right)+\varepsilon\left\lceil C_{0}\left(\delta_{0}\right)\right\rceil \geq \varepsilon\left(\frac{u_{0}(x)}{\varepsilon}-C_{0}\left(\delta_{0}\right)\right)+\varepsilon\left\lceil C_{0}\left(\delta_{0}\right)\right\rceil \geq u_{0}(x) .
$$

Let us now verify the equation. Since the equation is invariant w.r.t. $\varepsilon \times$ integer additions we only need to prove that

$$
\phi^{\varepsilon}(t, x):=\varepsilon h\left(\frac{\phi(t, x)}{\varepsilon},\left(u_{0}\right)_{x}(x)\right) \quad \text { with } \quad \phi(t, x):=u_{0}(x)+C t
$$


is a supersolution of (1.5). Actually, all the computations have already been made during the proof of Lemma 3.2. We see that $\phi^{\varepsilon}$ is of the form (3.3) with $v \equiv 0$. Moreover, $\phi$ satisfies (3.5) with $\delta:=\delta_{0}$ and

$$
C_{\phi}:=\left\|\left(u_{0}\right)_{x}\right\|_{\infty}+\left\|\left(u_{0}\right)_{x x}\right\|_{\infty}+\left\|\left(u_{0}\right)_{x x x}\right\|_{\infty}, \quad \phi_{t}=C \quad \text { and } \quad \phi_{x t}=0 .
$$

We then argue exactly as in the proof of Lemma 3.2 to show (3.20), which in this case is:

$$
\mathcal{L}_{\varepsilon}\left[\phi^{\varepsilon}\right]=h_{\tilde{z}} C-h_{\tilde{z}} K A \phi_{x x}+o(1)
$$

(since $v_{z}$ also equals zero). Therefore, we have

$$
\mathcal{L}_{\varepsilon}\left[\phi^{\varepsilon}\right]=h_{\tilde{z}}\left(\tilde{z}, \phi_{x}(t, x)\right) C-h_{\tilde{z}}\left(\tilde{z}, \phi_{x}(t, x)\right) K\left(\tilde{z}, \phi_{x}(t, x)\right) A\left(\tilde{z}, \phi_{x}(t, x)\right) \phi_{x x}(t, x)+o(1) .
$$

where $\lim _{\varepsilon \rightarrow 0} o(1)=0$ uniformly in $(t, x) \in(0, \infty) \times \mathbb{R}$. By Lemma 3.1, it is clear that there exists a constant $M$ (not depending on $C$ ) such that for all $\varepsilon$ small enough, $\mathcal{L}_{\varepsilon}\left[\phi^{\varepsilon}\right] \geq m\left(\delta_{0}\right) C-M$ on $(0, \infty) \times \mathbb{R}$. We conclude that $u^{+, \varepsilon}$ is a supersolution of $(4.1)$ for $C \geq \frac{M}{m\left(\delta_{0}\right)}$.

The same way, we prove that $u^{-, \varepsilon}$ is a subsolution of (4.1). By the comparison principle, we deduce that $u^{-, \varepsilon} \leq u^{\varepsilon} \leq u^{+, \varepsilon}$. Moreover, it is easy to show from Lemma 3.1 item (a) that

$$
\left|\varepsilon h\left(\frac{u_{0}(x) \pm C t}{\varepsilon},\left(u_{0}\right)_{x}(x)\right)-\left(u_{0}(x) \pm C t\right)\right| \leq \varepsilon C_{0}\left(\delta_{0}\right),
$$

for all $(t, x) \in(0, \infty) \times \mathbb{R}$ and $\varepsilon>0$. It follows that $\left|u^{ \pm, \varepsilon}(t, x)-\left(u_{0}(x) \pm C t\right)\right| \leq 2 \varepsilon\left\lceil C_{0}\left(\delta_{0}\right)\right\rceil$.

To conclude, we have proved that there exists a positive constant $C$ such that for all $\varepsilon$ small enough and all $(t, x) \in(0, \infty) \times \mathbb{R}$,

$$
\left|u^{\varepsilon}(t, x)-u_{0}(x)\right| \leq C t+2 \varepsilon\left\lceil C_{0}\left(\delta_{0}\right)\right\rceil,
$$

where $\delta_{0}$ is the bound in (Ho) and $C_{0}\left(\delta_{0}\right)$ the bound in Lemma 3.1 (a), and this guarantees us the existence of the desired $\varepsilon$-uniform barriers on $u^{\varepsilon}$.

Step 2. Uniform bounds on the gradients. Using again the invariance of the PDE in (4.1) w.r.t. $\varepsilon \times$ integer additions, we see that (for fixed $a>0$ and $\varepsilon>0$ ) the functions $u^{\varepsilon}(t, x)+\varepsilon\left\lfloor\frac{\delta_{0} a}{\varepsilon}\right\rfloor$ and $u^{\varepsilon}(t, x)+\varepsilon\left[\frac{a}{\delta_{0} \varepsilon}\right\rceil$ are solutions of $(4.1)$ in $(0, \infty) \times \mathbb{R}$. But, hypothesis (Ho) implies for all $x \in \mathbb{R}$,

$$
u_{0}(x)+\varepsilon\left\lfloor\frac{\delta_{0} a}{\varepsilon}\right\rfloor \leq u_{0}(x+a) \leq u_{0}(x)+\varepsilon\left\lceil\frac{a}{\delta_{0} \varepsilon}\right\rceil
$$

at the initial time. By the comparison principle, we deduce that for all $(t, x) \in[0, \infty) \times \mathbb{R}$,

$$
u^{\varepsilon}(t, x)+\varepsilon\left\lfloor\frac{\delta_{0} a}{\varepsilon}\right\rfloor \leq u^{\varepsilon}(t, x) \leq u^{\varepsilon}(t, x)+\varepsilon\left\lceil\frac{a}{\delta_{0} \varepsilon}\right\rceil .
$$

Thus, letting $\varepsilon \rightarrow 0$ we obtain the bound on the gradient for the limits, i.e. $\frac{1}{\delta} \geq(\bar{u})_{x} \geq \delta$ and $\frac{1}{\delta} \geq(\underline{u})_{x} \geq \delta$. 
Step 3. Proof that $\bar{u}$ is a subsolution of (1.7). First note that the initial condition is trivially satisfied because of (4.2). We will argue now by contradiction. So let us assume that there exists $\phi \in C^{\infty}((0, \infty) \times \mathbb{R})$ such that $\bar{u}-\phi$ has a strict local maximum at $\left(t_{0}, x_{0}\right)$ with

$$
\phi_{t}\left(t_{0}, x_{0}\right)=\phi_{x x}\left(t_{0}, x_{0}\right) G\left(\phi_{x}\left(t_{0}, x_{0}\right)\right)+\eta
$$

for some $\eta>0$. Adding a constant to $\phi$ if necessary, one can assume that

$$
u^{0}\left(t_{0}, x_{0}\right)=\phi\left(t_{0}, x_{0}\right),
$$

so that

$$
u^{0}(t, x)<\phi(t, x) \quad \text { for all }(t, x) \neq\left(t_{0}, x_{0}\right) \text { sufficiently close to }\left(t_{0}, x_{0}\right) .
$$

In order to construct our perturbed test function, we choose $p_{0}=\phi_{x}\left(t_{0}, x_{0}\right)$ and $M_{0}=\phi_{x x}\left(t_{0}, x_{0}\right)$ in Proposition 1.3 and thus obtain the existence of $h, v$ and a unique real number $\lambda$ fulfilling (1.11). Notice that Step 2 ensures that $p_{0}>0$. Next, we define $\phi^{\varepsilon}$ as in $(3.3)$; by Lemma $3.2, \phi^{\varepsilon}(t, x)$ is well-defined for $(t, x, \varepsilon)$ sufficiently closed to $\left(t_{0}, x_{0}, \varepsilon\right)$. Our aim is to prove now that for $\varepsilon$ and $r>0$ small enough, $\phi^{\varepsilon}$ is a supersolution of (4.1) in a open set $Q_{r, r}:=Q_{r, r}\left(t_{0}, x_{0}\right)$ with $r$ independent of $\varepsilon$. By estimate (3.4) of Lemma 3.2, our thesis will be

$$
0 \leq \mathcal{L}_{\varepsilon}\left[\phi^{\varepsilon}\right](t, x)=h_{\tilde{z}}(\tilde{z}, p)\left[\phi_{t}(t, x)-\lambda+\mathcal{E}(t, x, \varepsilon)\right] \quad \forall(t, x) \in Q_{r, r},
$$

where $\tilde{z}=\frac{\tilde{\phi}^{\varepsilon}(t, x)}{\varepsilon}, p=\phi_{x}\left(t_{0}, x_{0}\right)$ and $z=\frac{\phi(t, x)}{\varepsilon}$. Since by (1.9) $h_{\tilde{z}}>0$, we are left to prove

$$
\phi_{t}(t, x)-\lambda+\mathcal{E}(t, x, \varepsilon) \geq 0 \quad \forall(t, x) \in Q_{r, r} \text { with } r \text { independent of } \varepsilon .
$$

We observe now that by the choice we made in Proposition 1.3, the real number $\lambda$ verifies $\lambda=$ $G\left(\phi_{x}\left(t_{0}, x_{0}\right)\right) \phi_{x x}\left(t_{0}, x_{0}\right)$. Therefore

$$
\begin{aligned}
\phi_{t}(t, x)-\lambda+ & \mathcal{E}(t, x, \varepsilon) \\
& =\phi_{t}(t, x)-\phi_{t}\left(t_{0}, x_{0}\right)+\phi_{t}\left(t_{0}, x_{0}\right)-\phi_{x x}\left(t_{0}, x_{0}\right) G\left(\phi_{x}\left(t_{0}, x_{0}\right)\right)+\mathcal{E}(t, x, \varepsilon) \\
& =\phi_{t}(t, x)-\phi_{t}\left(t_{0}, x_{0}\right)+\eta+\mathcal{E}(t, x, \varepsilon),
\end{aligned}
$$

where we used also (4.3). Since $\phi_{t}$ is continuous and $\lim _{(t, x, \varepsilon) \rightarrow\left(t_{0}, x_{0}, 0\right)} \mathcal{E}(t, x, \varepsilon)=0$, (4.6) follows. At this stage, we have proved that there exists $r>0$ such that for all $\varepsilon$ small enough, $\phi^{\varepsilon}$ is a supersolution of (4.1) in $Q_{r, r}$.

By Lemma 3.3, one can take a smaller $r>0$ if necessary to get the uniform convergence of $\phi^{\varepsilon}$ toward $u^{0}$ on $Q_{r, r}$. The same way, by (4.5), one can assume that

$$
\bar{u}(t, x) \leq \phi(t, x)-2 \theta \text { on } \overline{Q_{r, 2 r}} \backslash Q_{r, r} \text { for some } \theta>0 ;
$$

hence, for $\varepsilon$ small enough we have

$$
\phi^{\varepsilon}(t, x) \geq u^{\varepsilon}(t, x)+\varepsilon\left\lfloor\frac{\theta}{\varepsilon}\right\rfloor \text { on } \overline{Q_{r, 2 r}} \backslash Q_{r, r}
$$

where we used also the definition of $\bar{u}$. Since $u^{\varepsilon}$ is a solution of (4.1) in particular in the open set $Q_{r, r}$ and thanks to the invariance w.r.t. $\varepsilon \times$ integer translation, $u^{\varepsilon}(t, x)+\varepsilon\left\lfloor\frac{\theta}{\varepsilon}\right\rfloor$ is still a solution in $Q_{r, r}$. 
Our aim is to apply now the comparison result on bounded sets (Theorem 2.4). By Remark 2.5 and (4.7), this can be done for $\varepsilon \leq\left(\frac{r}{m}\right)^{\frac{1}{\alpha}}$ (i.e. $2 r \geq r+\varepsilon^{\alpha} m$ ). Thus

$$
\phi^{\varepsilon}(t, x) \geq u^{\varepsilon}(t, x)+\varepsilon\left\lfloor\frac{\theta}{\varepsilon}\right\rfloor \text { in all } Q_{r, r} .
$$

Letting $\varepsilon$ going to 0 we are led to $\phi(t, x) \geq \bar{u}(t, x)+\theta$ in $Q_{r, r}=Q_{r, r}\left(t_{0}, x_{0}\right)$ which evaluated at $\left(t_{0}, x_{0}\right)$ is in contradiction with (4.4). Therefore $\bar{u}$ is a subsolution of (1.7).

\section{Existence and main properties of $h, v, G$}

Let us now prove Proposition 1.3 and Proposition 1.4 that have been admitted before. We also prove the monotonicity of $G$ for the classical FK model. Proposition 1.3 is proved in Subsection 5.2 just after the proof of some preliminaries in Subsection 5.1. Propositions 1.4-1.5 are proved in Subsection 5.3. The last subsection is devoted to the proofs of some technical facts.

\subsection{Preliminaries on $f$}

The following result establishes the well-definition of $f$ defined by (1.2). This function is introduced in order to study problem (1.9) that defines the hull function $h$. Indeed, as we shall see in the next subsection, this will allow to rewrite (1.9) in the "more readable" form (5.5) which can be solved by the classical separation variable method.

Lemma 5.1. (Well-definition and main properties of $f$ )

Assume (H1) (i)-(v). Then, for each $h \in \mathbb{R}$, there exists a unique real $f(h)$ such that

$$
F\left(\left[-\frac{j^{2}}{2} f(h)\right]_{j}^{m}, h\right)=0 .
$$

Moreover, the function $f: \mathbb{R} \rightarrow \mathbb{R}$ thus defined is $C^{1}$ and 1-periodic.

Proof. Step 1: well-definition of $f$. Let us define the function $H=H(r, h)$ by

$$
H(r, h):=F\left(\left[-\frac{j^{2}}{2} r\right]_{j}^{m}, h\right) \text { for }(r, h) \in \mathbb{R}^{2} .
$$

For fixed $h \in \mathbb{R}$, consider the equation in $r$ :

$$
H(r, h)=F\left(\left[-\frac{j^{2}}{2} r\right]_{j}^{m}, h\right)=0 .
$$

By (H1) (i) and (v), $H \in C^{1}\left(\mathbb{R}^{2}\right)$ and satisfies:

$$
\frac{\partial H}{\partial r}(r, h)=-\sum_{1 \leq|i| \leq m} \frac{i^{2}}{2} F_{i}\left(\left[\frac{j^{2}}{2} r\right]_{j}^{m}, h\right) \leq-\nu<0 \quad \text { for all } \quad(r, h) \in \mathbb{R}^{2} .
$$

Equation (5.1) thus admits a unique solution $r:=f(h)$. 
Step 2: 1-periodicity. The function $H$ is in fact 1-periodic w.r.t. the $h$-variable, thanks to the periodicity of $F$ in (H1) (ii). It follows that for all $h \in \mathbb{R}$, we have

$$
H(f(h+1), h+1)=0=H(f(h), h)=H(f(h), h+1)
$$

and the uniqueness of the solution to (5.1) implies that $f(h+1)=f(h)$.

Step 3: regularity. By (5.2), the regularity result of the implicit function theorem implies that $f$ defined by (5.1) has (at least) the same regularity than $H$. We conclude that $f \in C^{1}(\mathbb{R})$ and complete the proof.

Let us remark that, as a consequence of Lemma 5.1, the hull function solving (1.9), satisfies in particular: $h_{z z}+\frac{1}{p^{2}} f(h)=0$. In order to solve this equation by the separation variable method, we have to introduce the function $\mathbb{K}=\mathbb{K}(p)$ defined by the lemma below. This result also states the main properties of $\mathbb{K}$ that will be needed for the qualitative study of $G$ in Subsection 5.3.

\section{Lemma 5.2. (Definition of $\mathbb{F}$ and $\mathbb{K}$ and main properties of $\mathbb{K}$ )}

Assume (H1) and let $\mathbb{F}$ be the 1-periodic primitive of $f$ with null mean. Then, for each $p>0$, there exists a unique real $\mathbb{K}(p)>\max \mathbb{F}$ such that

$$
\int_{0}^{1} \frac{d h}{\sqrt{2(\mathbb{K}(p)-\mathbb{F}(h))}}=\frac{1}{p}
$$

Moreover, the function $\mathbb{K}:(0, \infty) \rightarrow(\max \mathbb{F}, \infty)$ thus defined is analytic and satisfies:

$$
\mathbb{K}(p) \sim_{p \rightarrow \infty} \frac{p^{2}}{2} .
$$

We skip the details of the proof of Lemma 5.2 which is an elementary result.

\subsection{Proof of Proposition 1.3}

We are now able to prove Proposition 1.3.

Proof of item (a). By Lemma 5.1, Problem (1.9) is equivalent to the following one: for all $(z, p) \in$ $\mathbb{R} \times(0, \infty)$,

$$
\left\{\begin{array}{l}
h_{z z}(z, p)+\frac{1}{p^{2}} f(h(z, p))=0 \\
h(z+1, p)=h(z, p)+1 \\
h_{z}(z, p)>0 \\
h(0, p)=0
\end{array}\right.
$$

where $f \in C^{1}(\mathbb{R})$ is 1 -periodic with null mean. Let us solve this equation by the help of the separation variable method.

Step 1: existence of the hull function. For $(h, p) \in \mathbb{R} \times(0, \infty)$, define

$$
\phi(h, p):=p \int_{0}^{h} \frac{d \tau}{\sqrt{2(\mathbb{K}(p)-\mathbb{F}(\tau))}},
$$


where $\mathbb{K}$ and $\mathbb{F}$ are defined in Lemma 5.2. Since $\mathbb{K}:(0, \infty) \rightarrow(\max \mathbb{F}, \infty)$ is analytic and $\mathbb{F} \in C^{2}(\mathbb{R})$, we have $\phi \in C^{3}(\mathbb{R} \times(0, \infty))$ with

$$
\frac{\partial \phi}{\partial h}(h, p)=\frac{p}{\sqrt{2(\mathbb{K}(p)-\mathbb{F}(h))}} \geq \frac{p}{\sqrt{2(\mathbb{K}(p)-\min \mathbb{F})}}>0 .
$$

For fixed $p>0$, the function $\phi(\cdot, p): \mathbb{R} \rightarrow \mathbb{R}$ is thus invertible, $C^{3}$ and its inverse is also $C^{3}$, thanks to the regularity result of the inverse function theorem. Let us denote this inverse by

$$
h(z, p):=(\phi(\cdot, p))^{-1}(z),
$$

for each $z \in \mathbb{R}$, and let us prove that this function is solution to (5.5).

First, we have

$$
h_{z}=\left(\frac{\partial \phi}{\partial h}\right)^{-1}=\frac{\sqrt{2(\mathbb{K}(p)-\mathbb{F}(h))}}{p}
$$

in particular, $\frac{h_{z}^{2}}{2} p^{2}=\mathbb{K}(p)-\mathbb{F}(h)$ and derivating one time w.r.t. $z$, we get $h_{z z} h_{z} p^{2}+f(h) h_{z}=0$. Since (5.9) implies that $h_{z}$ is positive, we can divide this equation by $h_{z}$ to conclude that $h$ satisfies the ODE of (5.5).

Moreover, the 1-periodicity of $\mathbb{F}$ and (5.3) imply obviously that $\phi(h+1, p)=\phi(h, p)+1$ for all real $h$. Taking the inverse, the function $h$ defined in (5.8) satisfies:

$$
h(z+1, p)=(\phi(\cdot, p))^{-1}(z+1)=(\phi(\cdot, p))^{-1}(z)+1=h(z, p)+1
$$

for all $z \in \mathbb{R}, p>0$. Finally, we have already seen that $h_{z}>0$ and it is clear that $h(0, p)=0$ for all $p>0$. Indeed, $\phi(0, p)=0$ and it follows that $h(0, p)=(\phi(\cdot, p))^{-1}(0)=0$. We conclude that $h$ is solution to (5.5) and a fortiori to (1.9).

Step 2: uniqueness of the hull function. Assume that $\tilde{h}$ is another solution. Then multiplying the first equation (1.9) by $\tilde{h}_{z}$ and integrating, we see that $\frac{1}{2}\left(\tilde{h}_{z}\right)^{2}+\frac{1}{p^{2}} \mathbb{F}(\tilde{h})=\frac{C}{p^{2}}$ and then

$$
\int_{0}^{1} \frac{d \tilde{h}}{\sqrt{2(C-\mathbb{F}(\tilde{h}))}}=\int_{0}^{1} \frac{d z}{p}
$$

which implies that $C=K(p)$ and then $\tilde{h}=h$.

Step 3: regularity of the hull function. For $(h, p, z) \in \mathbb{R} \times(0, \infty) \times \mathbb{R}$, define $\psi(h, p, z):=$ $\phi(h, p)-z$. By $(5.8), h(z, p)$ is the unique real that satisfies the equation

$$
\psi(h(z, p), p, z)=0 .
$$

Moreover, $\psi \in C^{3}(\mathbb{R} \times(0, \infty) \times \mathbb{R})$ since $\phi$ is $C^{3}$, and (5.7) implies that $\frac{\partial \psi}{\partial h}(h, p, z)=\frac{\partial \phi}{\partial h}(h, p)>0$. By the regularity result of the implicit function theorem, we deduce that $h \in C^{3}(\mathbb{R} \times(0, \infty))$. The proof of the item (a) of Proposition 1.3 is now complete. 
Proof of items (b) and (c). Let $p_{0}>0$ and $M_{0} \in \mathbb{R}$ be fixed. We begin by rewriting equation (1.11) in the more "readable" form (5.10) below. To do this, observe that $K$ defined by (1.10) is positive, thanks to (H1) (v); since $h_{z}^{2}$ is also positive, the ODE in (1.11) is equivalent to

$$
\lambda \frac{h_{z}^{2}}{K}=M_{0} A h_{z}^{2}+p_{0}^{2}\left(\frac{h_{z}^{2}}{2} v_{z z}+h_{z z} h_{z} v_{z}\right) .
$$

Using now that $\left(\frac{h_{z}^{2}}{2} v_{z z}+h_{z z} h_{z} v_{z}\right)=\left(\frac{h_{z}^{2}}{2} v_{z}\right)_{z}$, we see that (1.11) is equivalent to

$$
\left\{\begin{array}{l}
\lambda \frac{h_{z}^{2}}{K}=M_{0} A h_{z}^{2}+\frac{p_{0}^{2}}{2}\left(\left(h_{z}\right)^{2} v_{z}\right)_{z} \\
v(z+1)=v(z)
\end{array}\right.
$$

We can now prove the existence and uniqueness of $\lambda$.

Step 1: uniqueness of $\lambda$. Assume that the equation above admits a solution $v \in C^{2}(\mathbb{R})$. Then, we can integrate (5.10) w.r.t. $z \in[0,1]$ and using the 1-periodicity of $h_{z}\left(\cdot, p_{0}\right)$ and of $v$, we get:

$$
\lambda \int_{0}^{1} \frac{h_{z}^{2}\left(z, p_{0}\right)}{K\left(z, p_{0}\right)} d z=M_{0} \int_{0}^{1} A\left(z, p_{0}\right) h_{z}^{2}\left(z, p_{0}\right) d z .
$$

This shows that there exists at most one $\lambda \in \mathbb{R}$ such that (5.10) admits a $C^{2}$ solution, and this $\lambda$ is given by (5.11).

Step 2: existence of $\lambda$. Conversely, assume that $\lambda$ satisfies (5.11) and let us prove that (1.11) has a solution. Define $H=H(z)$ by

$$
H:=\frac{2}{p_{0}^{2}}\left(\lambda \frac{h_{z}^{2}}{K}-M_{0} A h_{z}^{2}\right)
$$

By the regularity of $h$ and assumption (H1) (i), $A$ and $K$ are at least $C^{0}$ w.r.t. $z$ and so is $H$. Let $\mathcal{H}=\mathcal{H}(z)$ be a primitive of $H$ such that

$$
\int_{0}^{1} \frac{\mathcal{H}(z)}{h_{z}^{2}\left(z, p_{0}\right)} d z=0
$$

Let $v=v(z)$ be a primitive of $\frac{\mathcal{H}}{h_{z}^{2}}$, which is then 1-periodic. Since $H$ is $C^{0}, v$ is $C^{2}$. Moreover, by construction, we see that $v$ satisfies the ODE of (5.10).

Step 3: conclusion. To summarize, we have proved that (5.10) admits a solution iff (5.11) holds true; since (5.11) is equivalent to (1.12), we have completed the proof of both items (b) and (c) of Proposition 1.3.

\subsection{Qualitative properties of $G$}

Let us now prove the properties of $G$ in Propositions 1.4-1.5.

Proof of Propositions 1.4-1.5. The proof is based on the following decomposition for $p>0$ :

$$
G(p):=\mathbb{G}(p) \mathbb{I}(p) \text { with } \mathbb{G}(p):=\frac{\int_{0}^{1} 2 A(z, p) h_{z}^{2}(z, p) d z}{\int_{0}^{1} h_{z}^{2}(z, p) d z} \text { and } \mathbb{I}(p):=\frac{1}{2} \frac{\int_{0}^{1} h_{z}^{2}(z, p) d z}{\int_{0}^{1} \frac{h_{z}^{2}(z, p)}{K(z, p)} d z} \text {. }
$$


For the sake of clarity, the main properties of $\mathbb{G}$ will be stated and proved in the next subsection (see Lemma 5.4).

Step 1: positivity and regularity. Recalling that $K$ is positive by $(\mathbf{H} \mathbf{1})(\mathbf{v})$, we see that $\mathbb{I}$ is positive. Therefore, Lemma 5.4 implies that $G=\mathbb{G I}$ is positive. Moreover, by the item (a) of Proposition 1.3 and (1.12), it is clear that $G \in C^{0}(0, \infty)$; notice that $G$ is only $C^{0}$, since $F$ is assumed to be only $C^{1}$ in (H1) (i). But, if $F$ is $C^{k+1}$, then $G$ is $C^{k}$. Indeed, the regularity result of the implicit function theorem applied in Step 3 (resp. Step 1) of the proof of Lemma 5.1 (resp. of the item (a) of Prop. 1.3), would imply that $f$ is $C^{k+1}$ (resp. that $h$ is $C^{k+3}$ ). Thus, $A$ and $K$ would be at least $C^{k}$ and $G$ also.

Step 2: limit as $p \rightarrow 0$. Because of (H1)(i) we remark that

$$
M:=\sup _{\mathbb{R} \times(0, \infty)} K<\infty .
$$

It follows that

$$
0<\mathbb{I} \leq \frac{M}{2} \frac{\int_{0}^{1} h_{z}^{2}(z, p) d z}{\int_{0}^{1} h_{z}^{2}(z, p) d z}=\frac{M}{2} .
$$

Since $\lim _{p \rightarrow 0} \mathbb{G}(p)=0$ by Lemma 5.4, we conclude that $\lim _{p \rightarrow 0} G(p)=0$.

Step 3: limit as $p \rightarrow \infty$. Let us study the limits, as $p \rightarrow \infty$, of the different terms that define $\mathbb{I}$.

First, it is clear that $h_{z z}(z, p) \rightarrow 0$ as $p \rightarrow \infty$, uniformly in $z \in[0,1]$. Moreover, (5.9) and (5.4) easily imply that $\lim _{p \rightarrow \infty} h_{z}(0, p)=1$. The primitives $h_{z}$ of $h_{z z}$ then have to satisfy:

$$
h_{z}(z, p) \rightarrow 1 \text {, as } p \rightarrow \infty \text {, uniformly in } z \in[0,1] .
$$

Next, (5.15) and the initial condition $h(0, p)=0$ imply that $h(z, p) \rightarrow z$, as $p \rightarrow \infty$, uniformly in $z \in[0,1]$. Consequently, $p^{2} h_{z z}(z, p)=-f(h(z, p)) \rightarrow-f(z)$ as $p \rightarrow \infty$, uniformly in $z \in[0,1]$. By the regularity of $F$ and (1.10), it is easy to deduce that

$$
K(z, p) \rightarrow K(z, \infty) \text { as } p \rightarrow \infty \text {, uniformly in } z \in[0,1],
$$

where

$$
K(z, \infty):=\sum_{1 \leq|i| \leq m} i^{2} F_{i}\left(\left[-j^{2} \frac{f(z)}{2}\right]_{j}^{m}, z\right) .
$$

By (H1) (i) and (v), the limit function $K(\cdot, \infty)$ is still positive and continuous on $\mathbb{R}$. Then, $\min _{[0,1]} K(\cdot, \infty)=: \gamma>0$, which implies that $\min _{z \in[0,1]} K(z, p) \geq \gamma / 2$ for $p$ sufficiently large; in particular, we get:

$$
\frac{1}{K(z, p)} \rightarrow \frac{1}{K(z, \infty)} \text { as } p \rightarrow \infty, \text { uniformly in } z \in[0,1] .
$$

Passing finally to the limit under the integral signs that defines $\mathbb{I}$ in (5.13), we deduce from (5.15)(5.17) that

$$
\lim _{p \rightarrow \infty} \mathbb{I}(p)=\frac{1}{2}\left\{\int_{0}^{1} \frac{1}{K(z, \infty)} d z\right\}^{-1}=l>0
$$


where $l$ is defined in (1.13). Since Lemma 5.4 states that $\lim _{p \rightarrow \infty} \mathbb{G}(p)=1$, we have proved that $\lim _{p \rightarrow \infty} G(p)=l$.

Step 4: analyticity and monotonicity for the classical FK model. For the classical FK model (1.3), simple computations show that $K(z, p)$ is constant equal to 2 . Hence, $\mathbb{I}=1$ and $G=\mathbb{G}$. The proof of the analyticity and monotonicity of $G$ is then an immediate corollary of Lemma 5.4.

\subsection{Technical results: properties of $\mathbb{G}$}

Let us first prove the following technical lemma.

Lemma 5.3. For $\alpha \in \mathbb{R}$ and $p>0$, define $J^{\alpha}(p):=\int_{0}^{1}(2(\mathbb{K}(p)-\mathbb{F}(h)))^{\alpha}$ dh, where $\mathbb{K}(p)$ is defined by (5.3). Then, $J^{\alpha}$ is positive, analytic w.r.t. $p>0$ and for $i \in \mathbb{Z}$,

$$
\begin{aligned}
\left(J^{\frac{i}{2}}\right)^{\prime} & =i \mathbb{K}^{\prime} J^{\frac{i-2}{2}}, \\
J^{\frac{i}{2}} & <\sqrt{J^{\frac{i+2}{2}} J^{\frac{i-2}{2}}} \quad \text { if } f \not \equiv 0, \\
J^{-\frac{1}{2}} & \leq\left(J^{-\frac{3}{2}}\right)^{\frac{1}{3}} .
\end{aligned}
$$

Proof. By Lemma $5.1, J^{\alpha}$ is analytic. Of course $J^{\alpha}$ is positive, since $\mathbb{K}>\max \mathbb{F}$. Moreover, Equality (5.18) is an immediate consequence of the theorem of derivation under the integral sign. To prove (5.19), we use Cauchy-Schwartz's inequality. We have

$$
\begin{aligned}
& \int_{0}^{1}(2(\mathbb{K}(p)-\mathbb{F}(h)))^{\frac{i}{2}} d h=\int_{0}^{1}(2\mathbb{K}(p)-\mathbb{F}(h)))^{\frac{i+2}{4}}(2(\mathbb{K}(p)-\mathbb{F}(h)))^{\frac{i-2}{4}} d h \\
& \leq \sqrt{\int_{0}^{1}(2(\mathbb{K}(p)-\mathbb{F}(h)))^{\frac{i+2}{2}} d h \times \int_{0}^{1}(2(\mathbb{K}(p)-\mathbb{F}(h)))^{\frac{i-2}{2}} d h}
\end{aligned}
$$

which proves that $J^{\frac{i}{2}} \leq \sqrt{J^{\frac{i+2}{2}} J^{-\frac{i-2}{2}}}$. But, these terms can not be equaled, because this would imply that

$$
\forall h \in[0,1], \quad(2(\mathbb{K}(p)-\mathbb{F}(h)))^{\frac{i+2}{4}}=C(2(\mathbb{K}(p)-\mathbb{F}(h)))^{\frac{i-2}{4}}
$$

for some constant $C$; since the primitive $\mathbb{F}$ of $f$ is non-constant, it is clear that such an equality does not hold true ${ }^{1}$. The same way, (5.20) follows by Hölder's inequality with exponents $(p, q)=(3 / 2,3)$ giving $J^{-\frac{1}{2}} \leq\left(J^{0}\right)^{\frac{2}{3}}\left(J^{-\frac{3}{2}}\right)^{\frac{1}{3}}$ and the proof is complete.

Lemma 5.4. Under (H1), $\mathbb{G}$ is analytic, positive and increasing on $(0, \infty)$. Moreover, we have $\lim _{p \rightarrow 0} \mathbb{G}(p)=0$ and $\lim _{p \rightarrow \infty} \mathbb{G}(p)=1$.

Proof. Step 1: new formula for $\mathbb{G}$. By the definitions of $\mathbb{G}$ and $A$ in (5.13) and (1.10), simple computations show that

$$
\mathbb{G}=\mathbb{G}(p)=1+p \frac{\int_{0}^{1} 2 h_{z p}(z, p) h_{z}(z, p) d z}{\int_{0}^{1} h_{z}^{2}(z, p) d z}=1+p \frac{\frac{d}{d p}\left(\int_{0}^{1} h_{z}^{2}(z, p) d z\right)}{\int_{0}^{1} h_{z}^{2}(z, p) d z}:=1+p \frac{I^{\prime}(p)}{I(p)},
$$

\footnotetext{
${ }^{1}$ Excepted in the trivial case $f \equiv 0$, which is not interesting in our settings.
} 
where we set $I:=I(p)=\int_{0}^{1} h_{z}^{2}(z, p) d z$.

Note that equation (5.9) implies that $I(p)=\int_{0}^{1} \frac{1}{p} \sqrt{2(\mathbb{K}(p)-\mathbb{F}(h(z, p))} h_{z}(z, p) d z=\frac{J^{\frac{1}{2}}(p)}{p}$, where the powers $J^{\alpha}$ are defined and studied in Lemma 5.3.

Let us compute $I^{\prime}$. By (5.18), it follows that $I^{\prime}=\frac{\mathbb{K}^{\prime} J^{-\frac{1}{2}}}{p}-\frac{J^{\frac{1}{2}}}{p^{2}}=\frac{\mathbb{K}^{\prime} J^{-\frac{1}{2}}}{p}-\frac{I}{p}$. Equation (5.21) thus gives

$$
\mathbb{G}=1+\mathbb{K}^{\prime} \frac{J^{-\frac{1}{2}}}{I}-1=\frac{p \mathbb{K}^{\prime} J^{-\frac{1}{2}}}{J^{\frac{1}{2}}} .
$$

But (5.3) implies that $J^{-\frac{1}{2}}=\frac{1}{p}$. Since $\left(J^{-\frac{1}{2}}\right)^{\prime}=-\mathbb{K}^{\prime} J^{-\frac{3}{2}}$, we deduce that

$$
\mathbb{K}^{\prime}=\frac{1}{p^{2} J^{-\frac{3}{2}}}=\frac{J^{-\frac{1}{2}}}{p J^{-\frac{3}{2}}}
$$

hence, we get the following formula on $\mathbb{G}$ :

$$
\mathbb{G}=\frac{\left(J^{-\frac{1}{2}}\right)^{2}}{J^{\frac{1}{2}} J^{-\frac{3}{2}}} .
$$

Step 2: positivity and analyticity of $\mathbb{G}$. By (5.23) and Lemma 5.3 below, it is readily seen that $\mathbb{G}$ is positive and analytic w.r.t. $p$.

Step 3: monotonicity of $\mathbb{G}$. To compute $\mathbb{G}^{\prime}$, we simply have to derivate (5.23). Using (5.18), we leave it to the reader to verify that this leads to the following formula:

$$
\mathbb{G}^{\prime}=\mathbb{K}^{\prime}\left\{3 \frac{\left(J^{-\frac{1}{2}}\right)^{2} J^{-\frac{5}{2}}}{J^{\frac{1}{2}}\left(J^{-\frac{3}{2}}\right)^{2}}-\frac{\left(J^{-\frac{1}{2}}\right)^{3}}{\left(J^{\frac{1}{2}}\right) 2 J^{-\frac{3}{2}}}-2 \frac{J^{-\frac{1}{2}}}{J^{\frac{1}{2}}}\right\} .
$$

On denoting the three terms in brackets by $I_{1}, I_{2}$ and $I_{3}$, we get $\mathbb{G}^{\prime}=\mathbb{K}^{\prime}\left(3 I_{1}-I_{2}-2 I_{3}\right)$. Let us prove that $I_{1}>I_{2}$ and $I_{1}>I_{3}$, thus concluding the positivity of $\mathbb{G}^{\prime}$, since $\mathbb{K}^{\prime}$ is positive by (5.22) and Lemma 5.3. To establish that $I_{1}>I_{2}$, we have to prove that

$$
\frac{\left(J^{-\frac{1}{2}}\right)^{3}}{\left(J^{\frac{1}{2}}\right)^{2} J^{-\frac{3}{2}}}<\frac{\left(J^{-\frac{1}{2}}\right)^{2} J^{-\frac{5}{2}}}{J^{\frac{1}{2}}\left(J^{-\frac{3}{2}}\right)^{2}}
$$

which is equivalent to $J^{-\frac{1}{2}} J^{-\frac{3}{2}}<J^{-\frac{5}{2}} J^{\frac{1}{2}}$. But (5.19) implies that $J^{-\frac{1}{2}}<\sqrt{J^{\frac{1}{2}} J^{-\frac{3}{2}}}$ and $J^{-\frac{3}{2}}<$ $\sqrt{J^{-\frac{1}{2}} J^{-\frac{5}{2}}}$. Taking the product, we get the result.

The same way, $I_{1}>I_{3}$ is equivalent to $\left(J^{-\frac{3}{2}}\right)^{2}<J^{-\frac{1}{2}} J^{-\frac{5}{2}}$, which is already given by (5.19).

Step 4: limits as $p \rightarrow 0, \infty$. By (5.4), we see that $J^{\alpha}(p) \sim_{p \rightarrow \infty} p^{2 \alpha}$. By (5.23), we deduce that $\lim _{p \rightarrow \infty} \mathbb{G}(p)=1$. To compute the limit as $p \rightarrow 0$, remark first that

$$
J^{\frac{1}{2}} \geq \int_{0}^{1} \sqrt{2(\max \mathbb{F}-\mathbb{F}(h))} d h=: C_{0}>0 .
$$

Next, recall that (5.3) implies $J^{-\frac{1}{2}}=\frac{1}{p}$. By (5.20), we get: $\left(J^{-\frac{3}{2}}\right)^{\frac{1}{3}} \geq \frac{1}{p}$. Finally, we deduce from $(5.23)$ that $\mathbb{G}(p) \leq \frac{p}{C_{0}}$ and thus $\lim _{p \rightarrow 0} \mathbb{G}(p)=0$. The proof of Lemma 5.4 is complete. 


\section{A The ansatz and the cell equation for $\alpha=2$}

Let us give the main lines of the proof of the convergence result in Theorem 1.6. One uses an ansatz of the same form than in (1.8),

$$
U^{\varepsilon}(t, x)=\varepsilon h\left(\frac{\tilde{U}^{\varepsilon}(t, x)}{\varepsilon}, \tilde{u}_{x}^{0}(t, x)\right) \quad \text { with } \quad \tilde{U}^{\varepsilon}(t, x):=\tilde{u}^{0}(t, x)+\varepsilon^{2} \tilde{v}\left(\frac{\tilde{u}^{0}(t, x)}{\varepsilon}\right),
$$

where the hull function is as before and the corrector $\tilde{v}$ is defined by the proposition below.

\section{Proposition A.1. (The new corrector and coefficient)}

Assume $(\mathbf{H 1})$. Let $h \in C^{3}(\mathbb{R} \times(0, \infty))$ be the hull function in Proposition 1.3. Let $A, K, G, \tilde{K}$ be defined in (1.10), (1.12) and (1.17). Then:

\section{(a) The new corrector $\tilde{v}$}

For all $p_{0}>0$ and $M_{0} \in \mathbb{R}$ there exists a unique $\tilde{\lambda} \in \mathbb{R}$ such that there exists $\tilde{v} \in C^{2}(\mathbb{R})$ satisfying for all $z \in \mathbb{R}$

$$
\left\{\begin{array}{l}
\tilde{\lambda}=\tilde{K}\left(z, p_{0}\right) \frac{h_{z z z}\left(z, p_{0}\right)}{h_{z}\left(z, p_{0}\right)} p_{0}^{3}+K\left(z, p_{0}\right) A\left(z, p_{0}\right) M_{0}+K\left(z, p_{0}\right) p_{0}^{2}\left(\frac{\tilde{v}_{z z}(z)}{2}+\frac{h_{z z}\left(z, p_{0}\right)}{h_{z}\left(z, p_{0}\right)} \tilde{v}_{z}(z)\right), \\
\tilde{v}(z+1)=\tilde{v}(z) .
\end{array}\right.
$$

\section{(b) The coefficient $b$}

Moreover, we have $\tilde{\lambda}=b\left(p_{0}\right) p_{0}^{3}+G\left(p_{0}\right) M_{0}$ where $b$ defined in (1.16) is continuous.

Proof of Proposition A.1. We follow the lines of the proof of items (b) and (c) of Proposition 1.3 in Subsection 5.2. Multipyling by $\frac{h_{z}^{2}}{K}>0$ and using that $\left(\frac{h_{z}^{2}}{2} \tilde{v}_{z z}+h_{z z} h_{z} \tilde{v}_{z}\right)=\left(\frac{h_{z}^{2}}{2} \tilde{v}_{z}\right)_{z}$, we see that the ODE in (A.2) is equivalent to

$$
\tilde{\lambda} \frac{h_{z}^{2}}{K}=\frac{\tilde{K}}{K} h_{z z z} h_{z} p_{0}^{3}+M_{0} A h_{z}^{2}+\frac{p_{0}^{2}}{2}\left(\left(h_{z}\right)^{2} \tilde{v}_{z}\right)_{z} .
$$

Integrating w.r.t. $z \in[0,1]$ and using the 1-periodicity of the functions, we see that this equation admits a 1-periodic solution only if

$$
\tilde{\lambda} \int_{0}^{1} \frac{h_{z}^{2}\left(z, p_{0}\right)}{K\left(z, p_{0}\right)} d z=p_{0}^{3} \int_{0}^{1} \frac{\tilde{K}\left(z, p_{0}\right)}{K\left(z, p_{0}\right)} h_{z z z}\left(z, p_{0}\right) h_{z}\left(z, p_{0}\right) d z+M_{0} \int_{0}^{1} A\left(z, p_{0}\right) h_{z}^{2}\left(z, p_{0}\right) d z .
$$

This condition is equivalent to $\tilde{\lambda}=b\left(p_{0}\right) p_{0}^{3}+G\left(p_{0}\right) M_{0}$ where $b$ is defined in (1.16) and $G$ as before. Conversely, if this condition holds true, then one takes $\tilde{v}=\tilde{v}(z)$ as a primitive of $\mathcal{H}=\mathcal{H}(z)$ such that $\mathcal{H}_{z}=H:=\frac{2}{p_{0}^{2}}\left(\tilde{\lambda} \frac{h_{z}^{2}}{K}-\frac{\tilde{K}}{K} h_{z z z} h_{z} p_{0}^{3}-M_{0} A h_{z}^{2}\right)$ and $\int_{0}^{1} \frac{\mathcal{H}(z)}{h_{z}^{2}\left(z, p_{0}\right)} d z=0$. It is clear that $b$ is continuous since $F$ is $C^{1}$ and $h$ is $C^{3}$.

With Proposition A.1 in hands, we have to justify our new ansatz (A.1) by proving that the key local error estimate in Lemma 3.2 still works. 
Lemma A.2. (New local error estimate) Assume (H1) and let $\alpha=2$. Then Lemma 3.2 holds true by replacing $(v, \lambda)$ by $(\tilde{v}, \tilde{\lambda})$ from Proposition A.1.

Proof of Lemma A.2. We use the same notations than in the proof of Lemma 3.2. We repeat the computations by doing a Taylor's expansion of the hull function of one more order. Let us mention that we do Taylor's expansions of the same order for all the other functions. Consequently, the rests will depend on the same constants than before excepted $C_{1}(\delta)$ in Lemma 3.1. This constant appeared when having applied the Taylor-Young formula of order 2 to the hull function. Now, one shall use the classical Taylor's formula of order 3 and the rest will depend on the modulus of continuity of third-order derivatives of $h$. Instead of item (b) of Lemma 3.1, we thus shall need the following property:

(A.3)

For all $\delta>0$, the third-order partial derivatives of $h$ are uniformly continuous on $\mathbb{R} \times\left[\delta, \frac{1}{\delta}\right]$.

The proof of (A.3) is immediate since $h$ is $C^{3}$ and 1-periodic plus linear w.r.t. its first variable. Let us also recall that (3.5) can be assumed without loss of generality, and we do so.

For the reader's convenience, let us recall the notations of the different variables: $\tilde{z}:=\frac{\tilde{\phi}^{\varepsilon}(t, x)}{\varepsilon}$, $z:=\frac{\phi(t, x)}{\varepsilon}, p:=\phi_{x}(t, x), \phi^{\varepsilon}(t, x)=\varepsilon h(\tilde{z}, p)$, and $\tilde{\phi}^{\varepsilon}(t, x)=\phi(t, x)+\varepsilon^{2} \tilde{v}(z)$. Up to (3.13) we do exactly the same expansions. That is to say, one has (3.12) and (3.13) that we rewrite below:

$$
\begin{aligned}
(\mathrm{A} .4) \frac{\tilde{\phi}^{\varepsilon}\left(t, x+j \varepsilon^{\alpha}\right)}{\varepsilon} & =\tilde{z}+j \varepsilon^{\alpha-1} p+j \varepsilon^{\alpha} \tilde{v}_{z} p+\frac{j^{2}}{2} \varepsilon^{2 \alpha-1}\left(\phi_{x x}+\tilde{v}_{z z} p^{2}\right)+o\left(\varepsilon^{2 \alpha-1}\right)=: \tilde{z}+\mathcal{E}_{1}, \\
\phi_{x}\left(t, x+j \varepsilon^{\alpha}\right) & =p+j \varepsilon^{\alpha} \phi_{x x}+o\left(\varepsilon^{\alpha}\right)=: p+\mathcal{E}_{2}
\end{aligned}
$$

(where we recall that $\frac{o\left(\varepsilon^{\beta}\right)}{\varepsilon^{\beta}} \rightarrow 0$ as $\varepsilon \rightarrow 0$ uniformly in $(t, x, j)$; see $(3.10)$ ). Now we make an expansion of order 3 for the hull function. One gets Equation (3.16) with a new term:

$$
\begin{aligned}
& h\left(\frac{\tilde{\phi}^{\varepsilon}\left(t, x+j \varepsilon^{\alpha}\right)}{\varepsilon}, \phi_{x}\left(t, x+j \varepsilon^{\alpha}\right)\right)-h(\tilde{z}, p)= \\
& =j\left\{h_{\tilde{z}}\left(\varepsilon^{\alpha-1} p+\varepsilon^{\alpha} \tilde{v}_{z} p\right)+\varepsilon^{\alpha} h_{p} \phi_{x x}\right\}+\varepsilon^{2 \alpha-2}\left\{\frac{j^{2}}{2} h_{\tilde{z} \tilde{z}} p^{2}\right\}
\end{aligned}
$$

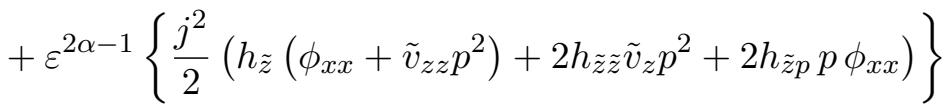

$$
\begin{aligned}
& +\underbrace{\varepsilon^{3 \alpha-3} \frac{1}{3 !} j^{3} p^{3} h_{\tilde{z} \tilde{z} \tilde{z}}}_{\text {new term }}+o\left(\varepsilon^{3 \alpha-3}\right),
\end{aligned}
$$

where the rest is controled by (A.3). Indeed, the rest is of order $o\left(\left(\left|\mathcal{E}_{1}\right|+\left|\mathcal{E}_{2}\right|\right)^{3}\right)$; moreover, recall that the worst term in $\left|\mathcal{E}_{1}\right|+\left|\mathcal{E}_{2}\right|$ comes from $j \varepsilon^{\alpha-1} p$ in (A.4) so that $\left(\left|\mathcal{E}_{1}\right|+\left|\mathcal{E}_{2}\right|\right)^{3}$ is at least of order $\varepsilon^{3 \alpha-3}$. 
Dividing by $\varepsilon^{2 \alpha-2}$, Equation (3.17) becomes:

$$
\begin{aligned}
& \frac{1}{\varepsilon^{2 \alpha-2}} V_{j}:=\frac{1}{\varepsilon^{2 \alpha-2}}\left\{h\left(\frac{\tilde{\phi}^{\varepsilon}\left(t, x+j \varepsilon^{\alpha}\right)}{\varepsilon}, \phi_{x}\left(t, x+j \varepsilon^{\alpha}\right)\right)-h(\tilde{z}, p)\right\} \\
& =j q+\frac{j^{2}}{2} h_{\tilde{z} z} p^{2}+\varepsilon j^{2} h_{\tilde{z}}\left\{A \phi_{x x}+p^{2}\left(\frac{\tilde{v}_{z z}}{2}+\frac{h_{\tilde{z} \tilde{z}}}{h_{\tilde{z}}} \tilde{v}_{z}\right)\right\} \\
& +\underbrace{\varepsilon^{\alpha-1} \frac{1}{3 !} j^{3} p^{3} h_{\tilde{z} \tilde{z} \tilde{z}}}_{\text {new term }}+o\left(\varepsilon^{\alpha-1}\right) .
\end{aligned}
$$

where $q:=\frac{1}{\varepsilon^{2 \alpha-2}}\left\{h_{\tilde{z}}\left(\varepsilon^{\alpha-1} p+\varepsilon^{\alpha} \tilde{v}_{z} p\right)+\varepsilon^{\alpha} h_{p} \phi_{x x}\right\}$ is the same linear displacement.

We proceed as in step 4 of the proof of Lemma 3.2 to remove the linear displacement. As in step 5, we make a Taylor's expansion of order 1 of $F$. Equation (3.19) becomes:

$$
\begin{aligned}
& \frac{1}{\varepsilon} F\left(\frac{1}{\varepsilon^{2 \alpha-2}}\left[V_{j}\right]_{j}^{m}, h\right)= \\
& =\frac{1}{\varepsilon} F\left(\left[\frac{j^{2}}{2} h_{\tilde{z} \tilde{z}} p^{2}\right]_{j}^{m}, h\right)+h_{\tilde{z}}\left\{K A \phi_{x x}+K p^{2}\left(\frac{\tilde{v}_{z z}}{2}+\frac{h_{\tilde{z} \tilde{z}}}{h_{\tilde{z}}} \tilde{v}_{z}\right)\right\}
\end{aligned}
$$

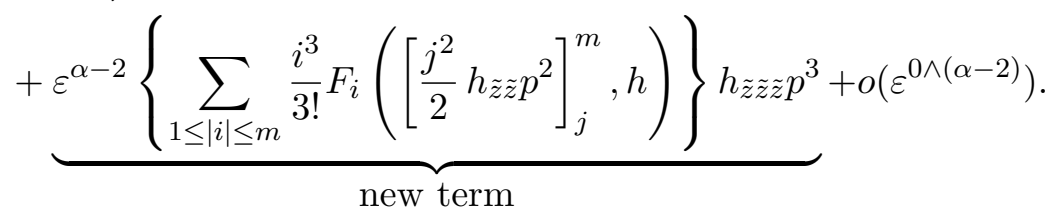

We recognize the function $\tilde{K}$ in (1.17). Equation (3.8) then becomes:

$$
\begin{aligned}
& \mathcal{L}_{\varepsilon}\left[\phi^{\varepsilon}\right]=h_{\tilde{z}} \phi_{t}+\varepsilon\left(h_{\tilde{z}} \tilde{v}_{z} \phi_{t}+h_{p} \phi_{x t}\right) \\
& -\frac{1}{\varepsilon} F\left(\left[\frac{j^{2}}{2} h_{\tilde{z} \tilde{z} p^{2}}\right]_{j}^{m}, h\right)-h_{\tilde{z}}\{\underbrace{\varepsilon^{\alpha-2} \tilde{K} \frac{h_{\tilde{z} \tilde{z} \tilde{z}}^{h_{\tilde{z}}}}{3}}_{\text {new term }}+K A \phi_{x x}+K p^{2}\left(\frac{\tilde{v}_{z z}}{2}+\frac{h_{\tilde{z} \tilde{z}}}{h_{\tilde{z}}} \tilde{v}_{z}\right)\}+o\left(\varepsilon^{0 \wedge(\alpha-2)}\right) .
\end{aligned}
$$

When $\alpha$ is strictly greater than 2 , the new term goes in the rest. Now it is of order $\varepsilon^{\alpha-2}=1$ and one has to take it into account in the equation of the corrector. To conclude, we proceed as in step 6 of the proof of Lemma 3.2 by using equation (A.2) to vanish the term of order 1.

We now leave it to the reader to verify that the convergence result in Theorem 1.6 can be proved the same way as in Section 4. The only difference is that one has to take into account the new term in (A.2) to construct the barriers in the first step; the new term that one has to control is of the form $\tilde{K}\left(\tilde{z}, \phi_{x}(t, x)\right) h_{\tilde{z} \tilde{z} \tilde{z}}\left(\tilde{z}, \phi_{x}(t, x)\right)$ where $\phi(t, x)=u_{0}(x)+C t$. Recalling that $F$ is $C^{1}$ and $h$ is $C^{3}$ and linear plus periodic w.r.t. its first variable, it is easy to control this term by (H0). Moreover, let us notice that the perturbed test function method uses a comparison principle for the limit equation as in Theorem 2.9. Since $b$ is continuous, it is classical that one has such a principle for $(1.15)$ (see [5]). 


\section{B An example of different limit when $\alpha=2$}

End of the proof of Theorem 1.6 We show that the new coefficient $b$ in (1.15) never equals zero. By (1.10) and (1.17), one easily computes that

$$
\frac{\tilde{K}}{K}=\frac{\left(l_{1}-l_{-1}\right)+8\left(l_{2}-l_{-2}\right)}{6\left(l_{1}+l_{-1}+4\left(l_{2}+l_{-2}\right)\right)}=: B \neq 0
$$

by assumption. By (1.16), one deduces that

$$
b(p)=B\left(\frac{\int_{0}^{1} h_{z z z}(z, p) h_{z}(z, p) d z}{\int_{0}^{1} \frac{h_{z}^{2}(z, p)}{K(z, p)} d z}\right)=-B\left(\frac{\int_{0}^{1} h_{z z}^{2}(z, p) d z}{\int_{0}^{1} \frac{h_{z}^{2}(z, p)}{K(z, p)} d z}\right),
$$

by integration by parts. Since $h$ is continuous and linear plus periodic and $F_{0}$ is continuous and non-constant, the intermediate value theorem implies that $F_{0}(h)$ is non-constant. Hence, by $(5.5)$, one deduces that $h_{z z}^{2}$ is non-constant and that $b(p) \neq 0$ for all $p>0$.

It remains to verify that (H1) holds true under (1.18). Item (v) is immediate. Let us show item (iv). By linearity of $F$ w.r.t. its first variable, one has to show that $\sum_{1 \leq|i| \leq 2} i l_{i}=0$, which is given by the condition $l_{1}+2 l_{2}=l_{-1}+2 l_{-2}$. The proof is complete.

\section{Acknowledgement}

The authors thank C. Imbert for fruitful discussions during the preparation of this work. This work was supported by the contract ANR MICA (2006-2009).

\section{References}

[1] G. BARLES, Some homogenization results for non-coercive Hamilton-Jacobi equations, Calc. Var. Partial Differential Equations 30 (2007), no. 4, 449-466.

[2] G. Barles, Solution de viscosité des équations de Hamilton-Jacobi, Springer Verlag, Paris, 1994.

[3] L. Boccardo, F. Murat, Remarques sur l'homogenisation de certaines problemes quasilineaires, Porugal. Math. 41 (1-4), 535-562 (1984).

[4] O.M. Braun, Y.-S. Kivshar, The Frenkel-Kontorowa Models, Concepts, Methods and Applications, Springer- Verlag, 2004.

[5] M.-G. Crandall, H. Ishit, P.-L. Lions, User's guide to viscosity solutions of second order partial differential equations, Bull. Amer. Math. Soc. (N.S.), 27, no. 2, (1992), pp.1-67.

[6] L.-C. Evans, The perturbed test function method for viscosity solutions of nonlinear PDE, Proc. Roy. Soc. Edimburgh Sect. A 111 (3-4), (1989), 359-375.

[7] L.-M. Floria, J.-J. Mazo, Dissipative dynamics of the Frenkel-Kantorova models, Advances in Physics 45 (6), (1996), 505-598. 
[8] N. Forcadel, C. Imbert, R. Monneau, Homogenization of Fully Overdamped FrenkelKontorova models, Journal of Differential Equations 246 (3), (2009), 1057-1097.

[9] N. Forcadel, C. Imbert, R. Monneau, Homogenization of some particle systems with two-body interactions and of the dislocation dynamics, Discrete and Continuous Dynamical Systems, 23 (3), (2009), 785-826.

[10] C. Imbert, R. Monneau, Homogenization of first-order equations with $(u / \varepsilon)$-periodic Hamiltonians. Part I: Local equations, Arch. Ration. Mech. Anal. 187 (2008), no. 1, 49-89.

[11] R. Jerrard, Singular limits of scalar Ginzburg-Landau equations with multiple-well potentials, Adv. Differential Equations 2 (1997), no. 1, 1-38.

[12] T. Kontorova, Y.-I. Frenkel, On the theory of plastic deformation and doubling (in Russian), Zh. Eksp. and Teor. Fiz. 8 (1938), 89-. , 1340-., 1349-.

[13] P.-L. Lions, G.-C. Papanicolau, S.-R.-S. Varadhan, Homogenisation of HamiltonJacobi equations, unpublished preprint, (1986). 